\title{
Polyvinylidene difluoride: A Universal Binder for Preparation of Solid Phase Microextraction Devices
}

\author{
Varoon Singh, Chiranjit Ghosh, Avneet Kaur, Janusz Pawliszyn*
}

${ }^{1}$ Department of Chemistry, University of Waterloo, 200 University Avenue West, Waterloo, Ontario N2L 3G1, Canada.

*Corresponding author

Prof. Janusz Pawliszyn

Department of Chemistry, University of Waterloo,

Ontario, N2L 3G1, Canada

Email: janusz@uwaterloo.ca

Phone: +1-519-888-4641

Fax: +1-519-888-4348

Present address

Varoon Singh

Laboratory of Chemical Analysis, Faculty of Veterinary Medicine, Ghent University, Salisburylaan 133, 9820 Merelbeke, Belgium Chiranjit Ghosh

Division of Infectious Diseases, Department of Medicine, Brigham and Women's Hospital and Harvard Medical School, Boston, Massachusetts, USA 


\begin{abstract}
The present communication describes the application of a fluorocarbon-based polymer as a high performance binder component for coatings suitable for a variety of solid phase microextraction (SPME) configurations. A polyvinylidene difluoride (PVDF) polymer was used to immobilize various sorbent particles on different supports to create different formats of SPME, namely fiber, thin-film membrane, and CBS devices. In this report, PVDF-based coatings are introduced as universal SPME coatings that are amenable to both gas chromatography (GC) and liquid chromatography (LC) while also improving the physical stability of the resulting device, in addition to eliminating the need for highly toxic reagents associated with the preparation of fully fluorinated based coatings previously reported in the literature. Additional incorporation of other polymers to increase coating porosity as well as the adhesion of PVDF on metal surfaces is also described.
\end{abstract}




\section{Introduction}

Binders play a key role in the preparation of solid phase microextraction (SPME) devices, ${ }^{1-4}$ exerting different effects on the extraction performance of said devices according to their ability to participate in the extraction process, while also influencing the extraction kinetics of the coating. Since the inception of SPME, ${ }^{5,6}$ much of the ensuing research in this area has been focused on the development of optimized sorbent materials that are able to outperform conventional sorbents, resulting in the development and application of materials such as Metal-organic frameworks (MOFs), Covalent-organic frameworks (COFs), Sol-gel, Silica, carbon nanotubes, graphene, and ionic liquids, among others for a variety of applications. However, while great strides have been made in the sorbent development area, significantly less effort has been dedicated to the development of optimized binders. Presently, polydimethylsiloxane (PDMS) ${ }^{1}$ and polyacrylonitrile (PAN) ${ }^{7}$ are most commonly used as binders for preparation of commercial SPME devices for GC and LC applications. Devices prepared using these binders have been frequently applied in environmental, food, and biological analyses for sampling and sample preparation due to the multiple advantages afforded by SPME, such as sampling and sample preparation in a single step, wide coverage of analytes, thermal/solvent desorption, high enrichment factors, biocompatibility, quick and rapid analysis, as well as low environmental impact. ${ }^{8,9}$ While the use of these binders has resulted in remarkably successful applications, neither of them can be used for both GC and LC applications together due to limitations associated with their physicochemical properties. PDMS is a highly viscous liquid that is thermally stable but susceptible to degradation in organic solvents and at extreme $\mathrm{pH}$ conditions. In addition, the use of PDMS necessitates long curing times during the preparation of coatings, as well as crosslinking to avoid monomer bleeding, which limits its use for GC applications. On the other hand, while PAN is a chemically stable, biocompatible solid binder with tunable viscosity, it is thermally labile and unstable at extreme alkaline $\mathrm{pH}$ conditions, restricting its use for preparation of GCamenable SPME devices. In analytical applications, the background of polymers used as binders and sorbents should be minimal so as to avoid contamination or instrument and signal suppression. Fabrication of SPME devices such as fibers, Thin Film Solid Phase microextraction (TF-SPME), and Coated Blade Spray (CBS) devices, among other formats, with the use of PDMS or PAN generally involves laborious procedures such as physical deposition, chemical bonding, gluing, dip coating, or bar coating. These methods are either manual in nature and/or require skilled manpower, resulting in procedures that are time consuming, expensive, and sometimes irreproducible. The objective of this work is to address these limitations with the introduction of fluorinated polymers as a binder for SPME coatings.

Fluorinated polymers ${ }^{10}$ have been widely used in a large variety of practical applications, such as thermal coatings to increase the environmental durability of paints on surfaces, as ultrafiltration membranes in water purification, ${ }^{11}$ as coatings in battery applications, ${ }^{12}$ as architectural coatings ${ }^{13,14}$ electrical insulation, piezoelectric films for switches, sensors, and loudspeakers, among their many uses, due to their exceptional thermal and chemical properties, good adhesion, superior chemical resistance, impact resistance, corrosion resistance, abrasion resistance, heat resistance, and good flexibility. ${ }^{15-18}$ Owing to their biocompatible nature, ${ }^{18}$ fluorinated polymers have also been used for preparation of devices for environmental, bioanalysis, and biomedical applications. Given the many beneficial properties afforded by fluorinated polymers, applications related to these polymers have been extraordinarily successful in material chemistry. In the area of analytical sample preparation, Nafion $^{\top \mathrm{M}}$, a sulfonated Teflon $^{\mathrm{TM}}$ copolymer having high conductivity, operating temperature, chemical stability, and biocompatibility, has been used as sorbent, ${ }^{19}$ binder $^{20}$ and as an antifouling coating ${ }^{21}$ for SPME and 
electrochemically enhanced SPME (EE-SPME) applications. Due to the presence of sulfonated groups and $-\mathrm{CF}_{2}$ groups, this material is both hydrophilic and hydrophobic in nature, and can thus help in the extraction of both types of analytes; however, it is also susceptible to solubilization in common solvents such as alcohol-water mixtures, ${ }^{22}$ making it susceptible to swelling, an undesirable property for SPME coatings. Most applications using Nafion have focused on sample preparation for GC, and this material has not been extensively explored for sample preparation for GC and LC applications together. When poly tetrafluoroethylene (Teflon) was used for GC and LC applications, superior results were obtained compared to commercial coatings (DVB/Car/PDMS fiber) for extraction of VOCs and SVOCs for GC and drugs of abuse from biofluids (C18/SCX/PAN fibers) for LC. ${ }^{23}$ However, the use of this material has limitations; for instance, it is only soluble in fluorinated solvents, which are highly toxic, volatile, and lead to ozone layer depletion. In addition, extraordinary measures are required to prepare glue or slurry from fluorinated solvents so as to avert changes in the viscosity and thickness of the resulting coating due to evaporation of the solvent at room temperature. As fluorinated polymers are solid binders that do not participate in extraction by facilitating adsorption of analytes in the layers of the coating due to their nonporous structure, unlike properties shown by the PDMS polymer, which is a viscous liquid binder, incorporation of higher boiling point fluorinated solvents as porogen in the glue has been suggested to both reduce the evaporation rate of the glue solvent as well as create pores in the coatings, which would lead to an increased sorption area, and thus facilitate extraction. ${ }^{23}$ Due to differences in boiling points, delayed evaporation of the porogen compared to the glue solvent creates pores that enable higher extraction efficiencies. In such cases, the second solvent should be fluorinated, as non-fluorinated and fluorinated solvents are not miscible due to hydrophobicity, and as a result do not address the Green Chemistry principles and evaporation issues typical of Teflon applications. Additionally, fluorinated polymers lack functional groups in their structure, making their adhesion on metal surfaces difficult. ${ }^{24}$ Such an issue is often addressed by undercoating the metal surface with another polymer or by etching the surface by electrolysis to make the surface of the metal very rough, steps that add complexity to the workflow while increasing the cost of the coating preparation procedure. When using an undercoating polymer, such a mixture should be thermally and chemically stable while not hindering the advantages afforded by the fluorinated polymer (no or minimal bleeding from sorbent and binder) for sample preparation applications involving mass spectrometers. Besides laboratory-scale applications, Teflon has not been used by the industry as an alternative for PDMS and PAN binders for SPME devices due to strict environmental regulations set for industry applications regarding the use of fluorinated solvents.

Poly vinylidene difluoride (PVDF), another fluorinated polymer, has been around for many years and like other fluorinated binders, has been used extensively for many applications. ${ }^{25}$ When compared to Teflon, PVDF as a binder is similar in structure except that it affords a higher amount of - $\mathrm{CH}$ bonds, a property that makes it superior as a binder for SPME applications. PVDF is thermally stable, chemically inert, and stable at 0-12 $\mathrm{pH}^{26}$ whereas crosslinked PVDF is stable from $0-14 \mathrm{pH},{ }^{26}$ soluble in solvents such as dimethyl sulfoxide, dimethylformamide, and $\mathrm{N}$-methyl pyrrolidone, as well as fluorinated solvents, while stable in general sample preparation solvents, ${ }^{27}$ making it a suitable binder for SPME applications. In analytical chemistry, PVDF has been exploited as a glue to adhere metal nanoparticles on fiber surfaces used for extraction of target analytes as well as to reduce background signals associated with functional groups present in other binders during analysis by Raman spectroscopy. ${ }^{28}$ Therefore, these leading properties of PVDF have motivated us to utilize it in the design of suitable analytical devices that can facilitate sample preparation methods employed in current analytical utilities. 
In this work, PVDF has been shown as a practical alternative to PDMS, PAN, Nafion ${ }^{\mathrm{TM}}$, and Teflon $^{\mathrm{TM}}$, thus addressing all the limitations associated with these binders. PVDF was used to prepare SPME fibers, TF-SPME membranes, and CBS devices using HLB, DVB, and nanocarboxen particles. The devices were assessed in terms of their physico-chemical properties, durability, and extraction performance. The effect of different molecular weights of the PVDF polymer on the extraction efficiencies of the prepared devices was also investigated. Polyvinylalcohol (PVA) and polyacrylonitrile were also added to coating mixtures to facilitate the creation of pores and to increase the adhesion of PVDF on metal surfaces, respectively. The performances of the created devices were evaluated by extracting McReynolds standards from the headspace of a standard gas generating vial ${ }^{29}$ for fiber and TF-SPME. For CBS devices, only the thermal and physical stability of the devices was investigated. A detailed analytical study including further investigations of the fashioned devices is expected in the near feature.

\section{Experimental Section}

Chemicals and Materials. Benzene, 2-pentanone, nitropropane, pyridine, 1-pentanol, octane, toluene, divinylbenzene, N-vinylpyrrolidone, 2-azobis(isobutyronitrile), Polyacrylonitrile (PAN, Mw 150000), polyvinylidene difluoride (PVDF, Mw: 180000 and 275000), polyvinyl alcohol (PVA), as well as the Commercial SPME fibers $100 \mu \mathrm{m}$ PDMS, fused silica, 65 $\mu \mathrm{m}$ PDMS/DVB, and Stableflex were bought from Millipore-Sigma (Mississauga, ON, Canada). HPLC-grade methanol, acetone, and acetonitrile were obtained from Caledon Laboratories Ltd. (Georgetown, ON, Canada). Ultrapure water (18.3 $\mathrm{M} \Omega \mathrm{cm}$ ) was obtained from a Barnstead/Thermodyne NANO-pure ultrapure water system (Dubuque, IA). DVB particles ( $5 \mu \mathrm{m}$ diameter) and high-density PDMS were provided by Supelco (Bellefonte, PA). The carbon fiber mesh weave (Panex 30) was provided by Zoltec Co. (Bridgetown, MO). Liquid nitrogen and ultrahigh-purity helium were supplied by Praxair (Kitchener, ON, Canada). The polystyrene-DVB resin (XAD-4) was provided by Sigma-Aldrich. HLB particles $(3-5 \mu \mathrm{m})$ were synthesized in-house. ${ }^{30}$ Super Activated Carbon Nanoparticles and Carbon Nanotubes Mixed (Nanocarboxen) were purchased from US research nanomaterials, Inc (Houston, Texas, USA). TF-SPME holding clips were supplied by GERSTEL Co. (Mülheim an der Ruhr, GE). A KJLC 704 silicon pump fluid (tetramethyl tetraphenyl trisiloxane) was ordered from Kurt J. Lesker Company (Toronto, ON, Canada). The membrane conditioning unit and electronic heating block used in this work were developed at the University of Waterloo Science Electronics Shop (Waterloo, ON, Canada). Cross-locking grip tweezers with stand were bought from KW surplus store (Kitchener, ON, Canada). Overhead stirrers with regulated speed controls were bought from Scilogex LLC (Rocky Hill, Connecticut, USA). The employed Elcometer 4340 motorized automatic film applicator and coating bar (adjustable gap of 0-250 $\mu \mathrm{m}$ ) were acquired from Elcometer Ltd. (Rochester Hills, MI). HLB-TF-SPME and DVB-TF-SPME membranes were prepared with PDMS as binder according to a method reported in the literature. ${ }^{1,2}$ Stainless steel blades were purchased from Shimifrez Inc. (Concord, Ontario, Canada). Blades (15 mm length) were coated either with HLB-polyacrylonitrile (HLB-PAN) or HLB-polyvinylidene difluoride (HLB-PVDF) slurries according to a protocol developed in our laboratory.

\section{Instrumental analysis method for the Benchtop GC-FID.}

An RTX-5 column (25 m x 0.25-mm i.d., 0.25- $\mu \mathrm{m}$ film thickness) from Restek (Bellafonte, PA, USA) was used for instrumental analysis. The carrier gas was helium, with a $1.2 \mathrm{~mL} \mathrm{~min}^{-1}$ flow rate; the injector head pressure was 9 psi. The injector port temperature was $250^{\circ} \mathrm{C}$, and the flame-ionization detector 
temperature was $280^{\circ} \mathrm{C}$. The oven temperature program was set as follows: $40^{\circ} \mathrm{C}$ for $1 \mathrm{~min}$, increased to $250^{\circ} \mathrm{C}$ at $25^{\circ} \mathrm{C} \mathrm{min}{ }^{-1}$, and held $0.75 \mathrm{~min}$.

Instrumental Analysis Method for the Benchtop GC-MS. An Agilent $6890 \mathrm{GC}$ and a 5973n quadrupole MS (Agilent Technologies, CA U.S.A.) were used for separation and quantitation, respectively. Sample introduction was achieved with a Gerstel MPS2 autosampler, which was used to transfer TF-SPME devices to the thermal desorption unit (TDU1) cooling injection system (CIS4) (GERSTEL, Mülheim an der Ruhr, GE) for membrane desorption. Chromatographic separations on the Agilent 6890-5973n were performed on a $30 \mathrm{~m} \times 0.25 \mathrm{~mm}$ I.D. $\times 0.25 \mu \mathrm{m}$ SLB-5 fused silica column (Millipore-Sigma, Mississauga, ON, CA). Helium served as carrier gas at a flow rate of $1.2 \mathrm{~mL} \mathrm{~min}^{-1}$. The column temperature was initially held at $40{ }^{\circ} \mathrm{C}$ for $2 \mathrm{~min}$, ramped to $140{ }^{\circ} \mathrm{C}$ at a rate of $8{ }^{\circ} \mathrm{C} \mathrm{min}{ }^{-1}$, then ramped to $250^{\circ} \mathrm{C}$ at $40^{\circ} \mathrm{C} \mathrm{min}^{-1}$, whereupon it was kept for $2 \mathrm{~min}$. The MS detector transfer line, MS quadrupole, and MS source temperatures were set at 300,150 , and $230^{\circ} \mathrm{C}$, respectively. Gas phase ions were generated using electron impact ionization at $70 \mathrm{eV}$, and the quadrupole was operated in SIM mode, selecting ions 78, 86, 43, 79, 55, and $85 \mathrm{~m} / \mathrm{z}$ for benzene, 2-pentanone, 1-nitropropane, pyridine,1-pentanol, and octane, respectively. To facilitate desorption from the $20 \mathrm{~mm} \times 4.75 \mathrm{~mm} \times 400 \mu \mathrm{m}(\mathrm{L} \times \mathrm{W} \times \mathrm{T})$ TF-SPME membranes, an inert glass bead was inserted into the tapered $5 \mathrm{~mm}$ I.D. glass desorption tube to prevent the membranes from slipping through the tapered bottom of the desorption tube, which was designed to hold a wider cylindrical PDMS stir bar rather than a flat thin film. Desorption was carried out at $250^{\circ} \mathrm{C}$ with a helium stripping gas flow of $60 \mathrm{~mL} \mathrm{~min}-1$ for $5 \mathrm{~min}$. The desorbed analytes were cryo-focused at $-130{ }^{\circ} \mathrm{C}$ within the $\mathrm{ClS}$ module. Following desorption, the $\mathrm{CIS}$ module was then ramped to a temperature of $270^{\circ} \mathrm{C}$ at a rate of $10^{\circ} \mathrm{C} \mathrm{s}-1$, to enable analyte transfer to the Agilent $6890 \mathrm{GC}$ column for separation and quantitation.

Characterization. The resulting morphologies, diameters, and porosities of the prepared SPME fibers, TFSPME membranes, and CBS devices were assessed using an Olympus SZX10 stereomicroscope system equipped with an SC30 digital camera (Olympus, Japan) and field emission scanning electron microscopy (FE-SEM Zeiss UltraPlus; Carl Zeiss Meditec AG, Jena, Germany) images. Samples were coated with a gold layer prior to FE-SEM analysis, and images were captured with the secondary electron detector at 4-10 kV $(1 \mathrm{~nm}$ resolution at $15 \mathrm{kV}$ ). The thermal stabilities of the fashioned SPME devices were assessed by comparing their background on GC-MS or GC-FID with commercial products at different temperatures. Thermogravimetric analyses of PVDF, HLB particles, and the PVDF/HLB blended coating were carried out prior to GC-MS or GC-FID analysis so as to avoid contamination of instruments. Thermograms were obtained via a TA Instruments Q500 Thermogravimetric analyser (TGA). Samples were heated under nitrogen in a platinum pan at $20 \mathrm{~K} \mathrm{~min}^{-1}$ from $50^{\circ} \mathrm{C}$ to $800^{\circ} \mathrm{C}$. The results pertaining to these analyses are discussed in the discussion section.

SPME Experiments. To evaluate the relative extraction efficiencies of fibers and TF-SPME devices prepared using the PVDF binder, commercial DVB/PDMS $(65 \mu \mathrm{m})$, PDMS $(100 \mu \mathrm{m})$, DVB/PDMS, and CAR/PDMS TF-SPME membranes were compared in terms of peak areas as discussed below. Extractions were performed from $20 \mathrm{~mL}$ and $250 \mathrm{~mL}$ McReynolds headspace generator jars prepared according to the method reported by Grandy et al. ${ }^{29}$ for fiber SPME and TF-SPME membranes, respectively. Headspace extractions were carried out at $55^{\circ} \mathrm{C}$ under static conditions. Analyte desorption was carried out for one minute at $280{ }^{\circ} \mathrm{C}$ for coated fiber, and for 6 minutes at $250{ }^{\circ} \mathrm{C}$ and $275^{\circ} \mathrm{C}$ for DVB/PVDF and NCAR/PVDF TF-SPME membranes, respectively. Consecutive fiber and membrane desorption blanks did not reveal the presence of analyte carryover on the coating, thus confirming the efficacy of the thermal desorption. All 
experiments were done in triplicate using three different membranes for TF-SPME. For fibers, inter-fiber reproducibility was assessed by using four fibers manufactured with slurries prepared on different days.

Coating suspension preparation For fiber. The biocompatible binder was prepared by dissolving appropriate amounts of PVDF and PVA in $\mathrm{N}, \mathrm{N}$-dimethylformamide (DMF) at an elevated temperature $\left(90^{\circ} \mathrm{C}\right)$. The solution was kept at this temperature and stirred every $15 \mathrm{~min}$ until it turned pale yellow in colour. After reaching room temperature, the glue was then mixed with sufficient particles to attain a $13.6 \%(\mathrm{w} / \mathrm{w})$ suspension. The suspension was sonicated using a probe sonicator for 2 minutes, then left overnight to stir at $900 \mathrm{rpm}$ to achieve homogeneous dispersion of particles in the glue. Following overnight stirring, the suspension was sonicated again, then used to coat the fibers.

For TF-SPME membranes. Glue was prepared by dissolving $12.5 \%$ of PVDF in $\mathrm{N}, \mathrm{N}$-dimethylformamide (DMF) at an elevated temperature $\left(90^{\circ} \mathrm{C}\right)$. The solution was kept at this temperature and stirred every 15 minutes until it turned pale yellow in colour. After reaching room temperature, sufficient particles were added to the glue to obtain a $5 \%(\mathrm{w} / \mathrm{w})$ suspension. The suspension was sonicated using a probe sonicator for 2 minutes, then stirred overnight at $900 \mathrm{rpm}$ to achieve homogeneous dispersion of particles in the glue. After overnight stirring, the suspension was sonicated again for $2 \mathrm{~min}$ right before use.

For Coated blade spray (CBS) devices. The binder for CBS devices was prepared by dissolving $12.5 \%(\mathrm{w} / \mathrm{v})$ of PVDF and an appropriate amount of PAN in DMF at an elevated temperature $\left(90^{\circ} \mathrm{C}\right)$. The mixture was then maintained at this temperature for $1 \mathrm{~h}$ and stirred every $15 \mathrm{~min}$, resulting in a pale yellow solution. After reaching room temperature, the glue was mixed with sufficient particles to obtain a $13.6 \%(w / w)$ suspension. The suspension was sonicated using a probe sonicator for 2 minutes, then left to stir overnight at $900 \mathrm{rpm}$ to achieve homogeneous dispersion of particles in the glue. After overnight stirring, the suspension was sonicated again prior to coating application.

\section{Preparation of SPME devices}

HLB/PVDF Fibers. Nitinol wires with a diameter of $200 \mu \mathrm{m}$ were abraded with sandpaper. Before use, sanded fibers were sonicated in water/methanol 50:50 (v/v) for 10 minutes and dried. The coating was deposited on the support by repeated dipping of $10 \mathrm{~mm}$ of the wire in a vial containing the extracting phase suspension at a speed of $5 \mathrm{~mm} / \mathrm{s}$ for dipping and $1 \mathrm{~mm} / \mathrm{s}$ for retracting, until the desired coating thickness was reached (total diameter of the coated tip was in the range of $100 \pm 5 \mu \mathrm{m}$ ). After each deposited layer, the coating was cured in an oven for $1 \mathrm{~min}$ at $125^{\circ} \mathrm{C}$, while the coating suspension was stirred at $600 \mathrm{rpm}$ to retain homogeneity and prevent phase separation. Depending on the viscosity of the slurry, different numbers of layers were applied to achieve similar thickness coatings. After the manufacturing procedure was concluded, SPME probes were submitted to soaking in a mixture of $\mathrm{MeOH} / \mathrm{ACN} / \mathrm{IPA}$ 50:25:25 (v/v/v) under vortex conditions at $1500 \mathrm{rpm}$ for $60 \mathrm{~min}$ to remove any residues that were acquired during the manufacturing process, such as uncured glue, monomers, and loose polymer particles. The prepared fibers were then mounted on hubs obtained from used commercial fibers. Fibers were thermally conditioned at $280^{\circ} \mathrm{C}$ for $2 \mathrm{~h}$ before first use.

Coated Blade Spray Devices. Stainless steel (SS) blades were acid etched for 30 minutes, washed with water, and cleaned in water/methanol 50:50 (v/v) for 10 minutes. After etching and cleaning, blades were dried for 20 minutes at $150{ }^{\circ} \mathrm{C}$ in an oven, then subsequently stored in a desiccator until their use. The coating was deposited on the support by repeated dipping of $10 \mathrm{~mm}$ of the SS blades in a vial containing the extracting phase suspension at speeds of $5 \mathrm{~mm} / \mathrm{s}$ for dipping and $1 \mathrm{~mm} / \mathrm{s}$ for retracting. While the 
process can be repeated if a thicker coating is desired, in this case, the fibers were coated by dipping only once (the total thickness of the coating on the SS blade tip was in the range of $100 \pm 5 \mu \mathrm{m}$ ). The coating was cured in an oven for $1 \mathrm{~min}$ at $125^{\circ} \mathrm{C}$, while the coating suspension was stirred at $600 \mathrm{rpm}$ to retain homogeneity and prevent phase separation. After the manufacturing procedure was concluded, CBS devices were subjected to cleaning in a mixture of $\mathrm{MeOH} / \mathrm{ACN} / \mathrm{IPA}$ 50:25:25 (v/v/v) under vortex conditions at $1500 \mathrm{rpm}$ for $15 \mathrm{~min}$ to remove any residues that were acquired during the manufacturing process, such as uncured glue, monomers, and loose polymer particles. The CBS devices were thermally conditioned before first use at $250^{\circ} \mathrm{C}$ for $12 \mathrm{~h}$ in a vacuum oven at a pressure of $15 \mathrm{mmHg}$.

\section{Particle Loaded TF-SPME Membranes.}

A $25 \mathrm{~cm} \times 60 \mathrm{~cm}$ (approx.) sheet of carbon mesh was cut and secured to the Elcometer 4340 motorized film applicator. The coating mixture was then manually placed in a thin strip along the top of the carbon mesh sheet. The coating bar gap was adjusted to the thinnest setting available, then used to slowly spread the sorbent mixture across the carbon mesh surface. The coating was then cured inside a nitrogen-purged vacuum oven at a pressure of $-15 \mathrm{mmHg}$ (approximately), and at $100^{\circ} \mathrm{C}$ for a period of at least $1 \mathrm{~h}$. As the membranes are double sided, the process was then performed a second time to complete the membrane. After coating both sides, membranes were cured at $250{ }^{\circ} \mathrm{C}$ for $12 \mathrm{~h}$. Next, individual membranes with dimensions of $2 \mathrm{~cm} \times 4.85 \mathrm{~mm}$ were manually cut from the prepared coating sheet so as to fit the Gerstel TDU. A brass template and sharp fabric cutting knife were used to make these cuts. It should be emphasized that it is essential that very clean cuts are employed when membranes are being sized; if not properly cut, loosely attached small strands of carbon may come loose during desorption and block the injector, thus compromising instrumental analysis. Membranes were conditioned under nitrogen at 250 ${ }^{\circ} \mathrm{C}$ for $4 \mathrm{~h}$ using a membrane conditioning unit developed in house by the University of Waterloo electronics shop. Once cooled, membranes were washed in a 25:25:25:25 water/methanol/ isopropanol/acetonitrile $v / v / v / v$ mixture for $2 \mathrm{~h}$ and then air-dried on Kimwipes. Before use, all membranes were submitted to a final 30 min conditioning step, with thermal desorption unit temperatures set at $250{ }^{\circ} \mathrm{C}$ for HLB and DVB membranes and $275^{\circ} \mathrm{C}$ for Nanocarboxen membranes. In line with standard SPME guidelines, it is recommended that this final conditioning step be performed again prior to use whenever the membranes have been stored for extended periods of time.

\section{Results and Discussion}

1. SPME sampling fiber with sorbent prepared by multiple dipping cycles

Nitinol wires of a $200 \mu \mathrm{m}$ diameter were roughened with sandpaper prior to coating. Following, the sanded fibers were sonicated in a solution of water/methanol 50:50 (v/v) for 10 minutes. A coating slurry was prepared by suspending $650 \mathrm{mg}$ of HLB particles (3-5 $\mu \mathrm{m}$ diameter) in a $6.5 \mathrm{~g}$ solution of PVDF in DMF.

Accordingly, the glue includes about $5 \mathrm{~g}$ of PVDF in $72 \mathrm{~mL}$ of DMF.

The slurry was deposited on the metallic support by dipping and slowly retracting the support from the slurry. Ten application cycles were used to prepare the extraction coating. After each cycle, the fiber was left in the $\mathrm{GC}$ oven to dry at $125^{\circ} \mathrm{C}$ for 1 minute. The resulting extraction coating was about $1 \mathrm{~cm}$ in length and $100 \mu \mathrm{m}$ in thickness, as shown in Fig. 1 . The thickness of the coating varied by less than $5 \%$ across the coated surface. No coating surface or morphology changes were observed following fiber exposure at 250 
${ }^{\circ} \mathrm{C}$ for 1 hour under inert atmosphere conditions, as can be seen in Fig. $2 \mathrm{a}$ and $2 \mathrm{~b}$, which respectively present optical microscope images of the fiber before thermal treatment and after thermal treatment at $250^{\circ} \mathrm{C}$.

2. SPME sampling fiber without sorbent prepared by multiple dipping cycles

Prior to the coating procedure, nitinol wires of a $200 \mu \mathrm{m}$ diameter were roughened with sandpaper and subsequently sonicated in a solution of water/methanol 50:50 (v/v) for 10 minutes. A coating slurry was prepared by dissolving 12\% PVDF (180K MW), 16\% PVDF (275K MW), 12.5\% PVDF (180K MW) with 6\% PVA, and 16\% PVDF (275K MW) with 2\% PVA in DMF (w/v). The fluorocarbon polymer was dissolved in the solvent by heating the mixture to $90^{\circ} \mathrm{C}$ for 1 hour while stirring at regular intervals.

The slurries were deposited on the metallic support by dipping then slowly retracting the support from the slurry. Fibers were coated several times till a thickness of $60 \mu \mathrm{m}$ was achieved. After each cycle, the fiber was left in the $\mathrm{GC}$ oven to dry at $125{ }^{\circ} \mathrm{C}$ for 1 minute. The resulting extraction coating was about 1 $\mathrm{cm}$ in length, as shown in Fig. 3. Fig. 3a , 3b, and 3c show fibers prepared using 12.5\% PVDF (180K MW), $16 \%$ PVDF (275K MW), and 16\% PVDF (275K MW) with 2\% PVA, respectively.

\section{SPME fibers prepared using different molecular weight fluorocarbon polymers}

The slurries were deposited on the metallic support by dipping then slowly retracting the support from the slurry. Fibers were submitted to this procedure several times until a coating thickness of $100 \mu \mathrm{m}$ was achieved. After each cycle, the fiber was left in the GC oven to dry at $125^{\circ} \mathrm{C}$ for 1 minute. The resulting extraction coating was about $1 \mathrm{~cm}$ in length. Fig. 4 shows scanning electron micrographs of fibers prepared using these slurries. Fig. 4a-c show fibers prepared using 12.5, 16 and 18\% PVDF (180K MW), whereas 4df show fibers prepared using 16,18 , and $20 \%$ PVDF (275K MW), with HLB particles (650mg 3-5 um diameter) suspended in each of them. Slurries with 10 and 25\% PVDF were either too viscous or fluid, making it difficult to coat them on supports to prepare fibers.

4. Preparation of porous SPME fiber using fluorocarbon polymer and non-fluorocarbon polymer

Prior to the coating procedure, nitinol wires with a dimeter of $200 \mu \mathrm{m}$ were roughened with sandpaper, then subsequently sonicated in a solution of water/methanol 50:50 (v/v) for 10 minutes. Eight distinct coating slurries were prepared by suspending HLB particles (3-5 um diameter) in $6.5 \mathrm{~g}$ solutions of PVDF (180K and $275 \mathrm{~K} \mathrm{MW}$ ) with varied percentages of PVA (2-8\%) in DMF. Four solutions of $12.5 \%$ PVDF (180K MW) and 2, 4,6, and 8\% PVA in DMF were prepared. The PVDF/PVA/DMF solutions were dissolved in a GC oven at $90^{\circ} \mathrm{C}$ for one hour, becoming homogenous and pale-yellow in colour. Similarly, 275K MW PVDF solutions containing either 2,4,6, and 8\% PVA were also prepared. The resulting solutions were used to prepare HLB/PVDF/PVA fibers.

The slurries were deposited on the metallic support by dipping slowly then retracting the support from the slurry. Fibers were coated several times till a coating thickness of $100 \mu \mathrm{m}$ was achieved. After each cycle, the fiber was left in the $\mathrm{GC}$ oven to dry at $125^{\circ} \mathrm{C}$ for 1 minute. The resulting extraction coating was about $1 \mathrm{~cm}$ in length. Fig. 5 shows scanning electron micrographs of fibers prepared using these slurries. Fig. 5a-c show fibers prepared using 12.5\% PVDF, $650 \mathrm{mg}$ HLB particles and 4, 6, and 8\% PVA while Fig. $5 \mathrm{~d}-\mathrm{f}$ show their expanded views. Fig. 6a-d show scanning electron micrographs of fibers prepared using $16 \%$ of PVDF with an average molecular weight of $275 \mathrm{~K}$ and $2,4,6$ and $8 \%$ PVA, while Fig. 6 e- $f$ show their expanded views. 
Fig. 7 presents the thermogravimetric curve of PVDF, indicating the very high thermal stability of the PVDF binder (approaching $500{ }^{\circ} \mathrm{C}$ ). The coating stability is limited by the thermal stability of the sorbent particles, in this case HLB.

5. Effect of ratio and chain length of fluorocarbon polymer on the extraction performance of SPME fiber Nitinol wires with a diameter of $200 \mu \mathrm{m}$ were coated with PVDF (180K and 275K MW) and of 12.5, 16, and $18 \%$ HLB particles.

A standard gas generating vial loaded with modified McReynold's standards was used to evaluate the extraction performance of the SPME fibers prepared using varying concentrations of fluorocarbon polymer and HLB particles. Extractions were performed using fibers shown in Figure 4 for 10 min while heating the standard gas generating vials at $45^{\circ} \mathrm{C}$. The gas generating vial was allowed to equilibrate for 10 minutes following each extraction.

The desorption, separation, and detection of analytes were performed on an HP 5890 GC-FID. The adsorbed analytes were thermally desorbed at $250^{\circ} \mathrm{C}$ for 2 minutes using ultrapure helium as a carrier gas and at a flow velocity of $1.5 \mathrm{ml} / \mathrm{min}$. The capillary column used for the chromatographic separation was an Agilent J\&W HP-5 (30m, $0.25 \mathrm{~mm}$ i.d., 0.25 um film thickness). Column temperature was initially set at $40{ }^{\circ} \mathrm{C}$ for 1 minute, then ramped at $10{ }^{\circ} \mathrm{C} / \mathrm{min}$ to $270^{\circ} \mathrm{C}$, where it was held for 2 minutes. Helium (purity level 99.9999\%) was used as carrier gas at a flow of $1.2 \mathrm{ml} / \mathrm{min}$.

The same sets of experiments and instrumental analyses were performed using an SPME sampling device equipped with a DVB/PDMS (65 um) coating in order to compare the extraction efficiencies of the prepared fibers at equilibrium conditions. When an SPME coating includes adsorptive particles, the adsorption mechanism of extraction requires that the extraction phase surface concentration of adsorbed analytes is considered rather than the extraction phase concentration. Therefore, calculations of fiber coating/sample distribution constants (Kfs) for such SPME adsorbents require that Se values be determined experimentally or that Se constants be known (since Se can be expressed as the ratio of amount extracted and the active surface of the fiber coating (Sa)). Since Sa is tedious to determine experimentally, a new constant, termed "fiber constant" (fc) representing the products Kfs*Sa for adsorptive materials, is alternatively used for estimations of SPME enrichment factors at equilibrium.

The DVB/PDMS (65 um) and PDMS (100um) coatings were obtained from Supelco of Millipore-Sigma. Extraction performance results are presented in FIG. 8 for the prepared fibers as compared to PDMS and DVB/PDMS fibers. Fibers prepared using 12.5\% PVDF (180K MW) and HLB particles yielded superior results when compared to other fibers prepared using $180 \mathrm{~K}$ and $275 \mathrm{~K}$ MW PVDF/HLB particles, except for octane. The extraction capability of $12.5 \%$ PVDF (275K MW) and DVB/PDMS was better for octane as compared to all other fibers. This reduced extraction capability of $12.5 \%$ PVDF (180K MW) for octane can be attributed to improper desorption or to the porosity of the SPME fiber. Considering the results, the $12.5 \%$ PVDF (180K MW) HLB fiber, which gave higher recoveries for other analytes except octane, was chosen for further testing. To increase the recovery of octane, desorption temperature was further studied.

6. Effect of desorption temperature on the recoveries of McReynolds compounds extracted using the SPME fiber

A standard gas generating (SGGV) vial loaded with modified McReynold's standards was used for the desorption temperature study. Extractions were performed in headspace mode using the $12.5 \%$ PVDF 
$(180 \mathrm{~K} \mathrm{MW}) / \mathrm{HLB}$ particles coated fiber. The fiber was conditioned in an inert atmosphere for $1 \mathrm{~h}$ at $280^{\circ} \mathrm{C}$, and blanks of the fibers were run before analysis. Analytes were extracted for 10 minutes from the SGGV heated at $45^{\circ} \mathrm{C}$, using the said fiber in triplicate. All instrumental parameters were kept as previously described except for desorption temperature. Analytes extracted by the new fiber were desorbed at 250 , 280,290 , and $300^{\circ} \mathrm{C}$, and the extraction performances were then compared to that of the DVB/PDMS fiber, which was desorbed at $250^{\circ} \mathrm{C}$. Fig. 9 shows that desorption temperature did not affect the response of the analytes and that all analytes were desorbed at $250^{\circ} \mathrm{C}$; yet, the response of the SPME fiber prepared using HLB/PVDF was not superior to that of the DVB/PDMS fiber for octane.

7. Effect of porosity on the extraction performance of the SPME fiber for McReynolds compounds

SPME fibers prepared in $\mathbf{4}$ were used for extraction of McReynolds compounds using SGGV as described in 5 . Analytes were extracted from the headspace of the vial for 10 minutes while the SGGV was heated at $45{ }^{\circ} \mathrm{C}$, then desorbed at $250^{\circ} \mathrm{C}$. It is evident from Fig. 10 that the SPME fibers prepared with 6\% PVA in 12.5\%PVDF and HLB particles yielded the best results for extraction of McReynolds compounds, whereas fibers prepared with lower molecular weight PVDF and higher percentage of PVA as porogen gave superior results for extraction of VOCs and SVOCs. In order to remove PVA from the fibers so as to create the pores, the prepared fibers were cleaned in water at $55^{\circ} \mathrm{C}$ for 30 minutes in a water bath.

\section{Extraction of McReynolds compounds using SPME fiber prepared using PVDF and PVDF/PVA}

The fibers prepared in $\mathbf{2}$ were used for extraction of McReynolds compounds. Extractions were carried out from the headspace of a SGGV heated at $45^{\circ} \mathrm{C}$ for 10 minutes. The extracted analytes were desorbed at $250^{\circ} \mathrm{C}$ in the injection port of the GC-FID. Fig. 11 shows the peak responses of all analytes as compared to those attained for the DVB/PDMS fiber. The data indicates that the PVDF polymer does not participate in the extraction of analytes, unlike PDMS, thus indicating that the extraction capacity of fibers prepared using PVDF as a binder is due to the adsorptive material only. Based on the data shown in Fig. 11 it can also be surmised that increasing the concentration of the PVDF polymer hinders the extraction performance of the fiber, whereas addition and subsequent removal of porogen shows remarkable improvement in extraction, particularly for heavier molecular weight compounds. This may be explained by the fact that PVDF is a solid binder and does not allow for absorption style extraction to occur throughout the volume of the PVDF as it typically occurs when PDMS is instead used in the preparation of SPME coatings. As a cross-linked viscous liquid, PDMS therefore enables absorptive extraction of analytes throughout its volume in a kinetically fast manner. The fact that PVDF is instead a solid polymer highlights the necessity of a porogen in the preparation of PVD- based extraction coatings, as these monolithic pores allow small organic compounds (such as the employed McReynolds probes) to directly interface with the immobilized sorbent particles. This direct sorbent-analyte contact via the monolithic pores eliminates the need for these analytes to diffuse through the solid volume of the PVDF binder, which would be kinetically very slow and impractical for SPME-style extractions. This described phenomena demonstrates significant novelty and significant improvement over previous attempts. Considering the effect of binder and porogen on the extraction performance of the developed SPME coatings, three fibers were selected for further investigation. SPME fibers prepared with 12.5\% PVDF and 6\% PVA with HLB particles, 12.5\% PVDF and $4 \%$ PVA with HLB particles, and 16\% PVDF and 2\% PVA with HLB particles. These fibers were compared in terms of their extraction capabilities, and as can be seen from Fig. 12, the fiber with 6\% PVA in 12.5\% PVDF and HLB particles outperformed commercial DVB/PDMS as well as the two other SPME fibers for 
extraction of target compounds. The carryover effect of the PVDF/PVA/HLB fibers was much lower or negligible when compared to the DVB/PDMS fiber.

9. Removal of porogen from the SPME fiber prepared using fluorocarbon polymer and non-fluorocarbon polymer with adsorptive materials

The fibers prepared in 4 were subjected to sonication while suspended in water in a vial at $55^{\circ} \mathrm{C}$ for 15 , $30,60,90$, and 120 minutes for removal of the porogen PVA, which once removed created monolithic pores on the prepared SPME fibers. Following PVA removal, fibers were cleaned again with water and then dried in a vacuum oven at $60^{\circ} \mathrm{C}$. Dried fibers were preconditioned at $280^{\circ} \mathrm{C}$ before extraction and a fiber blank was run in order to confirm the absence of interference peaks due to the PVA polymer. Fig. 13 shows extraction efficiency data for fibers prepared using HLB particles, 16\% PVDF (275K MW), and 2\% PVA. Fibers sonicated for 30 minutes were found to yield the best recoveries, with further prolonging of the sonication process exerting an adverse effect on the extraction performance of the fiber. This was due to a loss of coating resulting from longer exposure to sonication, which caused the fibers to lose some of their particles and binder as compared to fibers sonicated for shorter durations. Similar trends were observed for fibers prepared using HLB particles, 12.5\% PVDF (180K MW), and 6\% PVA, as can be seen in Fig. 14. Fibers sonicated for 15 minutes yielded superior results as compared to commercial DVB/PDMS and fibers sonicated for longer durations.

10. Inter-fiber reproducibility of SPME fiber prepared using HLB particles embedded in fluorocarbon polymer

Different batches of the fiber selected in 7 were prepared so as to evaluate inter-fiber reproducibility. Fibers were subjected to 10 min extractions of McReynolds compounds from the headspace of an SGGV heated to $45^{\circ} \mathrm{C}$. The extracted analytes were desorbed in the injection port of GC-FID for 2 minutes at $250^{\circ} \mathrm{C}$ and peak responses were compared to those of DVB/PDMS. The inter-fiber reproducibility of the fiber preparation procedure described in $\mathbf{4}$ was evaluated by preparing four different batches using the same composition of slurries prepared on different days. Fig. 15 shows that there was no remarkable difference in the extraction performance of the fibers made in different days for extraction efficiency of modified McReynold's compounds.

11. SPME sampling fabric prepared by film application

Coating slurries was prepared as described in 1. An untreated carbon mesh fabric having a unit weight of $115 \mathrm{~g} / \mathrm{m}^{2}$, a thickness of 406 micros, and a carbon content of $99 \%$ was immobilized on the surface of a bar coater in order to keep the fabric straight on the surface. The immobilized fabric was coated with the prepared slurry using a film applicator at a constant spreading speed so as to attain a carbon mesh fabric with a thin homogeneous coating layer. The coated fabric was left to dry in a vacuum oven at $200^{\circ} \mathrm{C}$ under nitrogen atmosphere for $2 \mathrm{~h}$ at a vacuum of -15 to -20 inch of $\mathrm{Hg}$, then allowed to cool before another coating was applied on the other side of the mesh. Given that the coating volumes and surface areas of SPME devices are greater when coated fabrics are used (thin-film format) as compared to coated wires (such as that used in 1), the authors of the present disclosure predicted that the prepared fabric would have a lower analyte detection threshold.

The slurries were prepared using commercial DVB particles obtained from Supelco, Sigma Aldrich, HLB particles (3-5 $\mu \mathrm{m}$ diameter) synthesized in house, Nanocarboxen obtained from US Nanomaterials 
research laboratory, and Carboxen particles from Supelco, Sigma Aldrich. FIG. 16 shows scanning electron micrographs of the bare carbon fabric (16a), DVB/PVDF/Carbon mesh (16b), HLB/PVDF/Carbon mesh (16c), and Nanocarboxen/PVDF/Carbon mesh. It is evident from these images that the particles are held in between the carbon fabric mesh by a very thin layer of binder on it. Due to the low viscosity of the coating mixture and the ease of application, a thin film with a higher particle loading can be obtained on the carbon fabric, which in turn can lower the analyte detection threshold. When compared to DVB/PDMS/Carbon mesh TFME, the background of siloxane can introduce issues in analysis as it may mask analytes of interest and sometimes hamper the detection of analytes at lower concentrations that yield lesser signal to noise ratios. The effect of siloxane was evaluated by running membrane blanks for the prepared membranes on an Agilent $6890 \mathrm{GC}$ and a 5973n quadrupole MS (Agilent Technologies, CA U.S.A.) for separation and quantitation, respectively. Sample introduction was achieved with a Gerstel MPS2 autosampler, which was used to transfer TF-SPME devices to the thermal desorption unit (TDU1) cooling injection system (CIS4) (GERSTEL, Mülheim an der Ruhr, GE) for membrane desorption. Chromatographic separations on the Agilent 6890-5973n were performed on a $30 \mathrm{~m} \times 0.25 \mathrm{~mm}$ I.D. $\times 0.25 \mu \mathrm{m}$ SLB-5 fused silica column (Sigma-Aldrich, Mississauga, ON, CA). Helium served as carrier gas at a flow rate of 1.2 $\mathrm{mL} / \mathrm{min}$. The column temperature was initially held at $40^{\circ} \mathrm{C}$ for $2 \mathrm{~min}$, ramped to $140{ }^{\circ} \mathrm{C}$ at a rate of $8{ }^{\circ} \mathrm{C}$ min-1, then ramped to $250^{\circ} \mathrm{C}$ at $40^{\circ} \mathrm{C}$ min-1, where it was kept for $2 \mathrm{~min}$. The MS detector transfer line temperature, MS quadrupole, and MS source temperatures were set at 300,150 , and $230^{\circ} \mathrm{C}$, respectively. Gas phase ions were generated using electron impact ionization at $70 \mathrm{eV}$, and the quadrupole was operated in Full scan mode to monitor the background of the membranes. Fig. 17 shows the backgrounds for DVB/PDMS membranes at the top, followed by DVB/PVDF, HLB/PVDF and Tube blank. It is evident that membranes prepared with PVDF as a binder did not yield any background peaks corresponding to the PVDF, whereas PDMS yielded a lot of siloxane peaks, which notably also appeared in the tube blank and in the chromatograms of the DVB/PVDF and HLB/PVDF membrane blanks. The only peaks observed that differed from those of the tube blank were detected around the retention time of 7 minutes. This background, which was also present in the chromatogram of DVB/PDMS membrane but seemingly suppressed by the siloxane peaks, could be attributed to the sorbent particles loaded onto the membranes, although these peaks were very small in chromatograms of HLB/PVDF membranes. It should also be noted that particles obtained from commercial sources should be thoroughly washed prior to use when making coating devices as they may contain residual monomers that could cause such peaks.

\section{SPME sampling blade prepared by dipping}

Probes may be coated according to the described procedure with a solid coating that generates ions when solvent and high voltage are applied. Such probes can be used for thermal or solvent desorption or for direct-to-mass-spectrometry applications where the coated probe will act as an ionization probe. Fig. 18 shows a bare metal surface(18a), a side view of the coated metal surface with fluorocarbon polymer and HLB particles (18b), the thickness of the coating on the surface of the metal probe (18c), a lateral view of the metal probe coated with 1 layer of fluorocarbon polymer/HLB particles (18d), and closer views of the binder and HLB particles on the surface of the metal probe (18e and f).

It is generally difficult to adhere fluorocarbon polymer on the surface of metals or alloys due a lack of functional groups that can form bonds with the surface and hold onto it. To avoid peeling of the fluorocarbon polymer and adsorptive material coating, another non-fluorocarbon polymer can be added to the slurry. This polymer can be any combination of polymers having functional groups other than fluorine in it or can be a co-polymer of the fluorinated polymer with another non-fluorinated polymer. For 
example, polyacrylonitrile can be added into this polymer slurry to adhere the coating to the surface of the metal. It can be added to the optimized slurry from $1-10 \%$ by weight of fluorocarbon polymer. It should be also ensured that the polymer does not degrade in the injection port of the GC, or yield any background signals when subjected to thermal or solvent desorption.

FIG. 19 shows the chromatograms of the backgrounds collected for blades prepared using the fluorocarbon polymer with HLB particles and a non-fluorinated polymer. Backgrounds were found to increase as the amount of non- fluorinated polymer added increased, showing that to some extent, when the non-fluorinated polymer is added to a fluorocarbon polymer, the resulting coating is capable of better withstanding heat, not showing observable effects in the total ion chromatogram (TIC) trace. However, increasing the concentration of non-fluorinated carbon or the number of layers of the coating can affect the background as well as the ability of the polymer to stay adhered to the metal surface. Peaks at retention times of 2 and 7 min correspond to the non-fluorinated polymer and HLB particles, respectively. Other peaks present in the chromatogram are due to the siloxane background coming from either the column or tubes. These peaks can also be seen in the blank chromatogram of the tube, shown as the bottom TIC trace in Fig 19.

\section{Conclusion}

The above examples clearly show that PVDF is a very useful binder that can be applied in a fashion similar to fully fluorinated binder ${ }^{23}$ for generation of thin robust SPME coatings that are suitable for both thermal and solvent desorption. Recently published work by others confirms this conclusion. ${ }^{31}$ Evaluations of these coatings for real matrices will be undertaken as a continuation of this work.

\section{Acknowledgements:}

Financial support was provided by Millipore Sigma and the Natural Sciences and Engineering Research Council of Canada under the Industrial Research Chair program.

\section{References:}

(1) Grandy, J. J.; Singh, V.; Lashgari, M.; Gauthier, M.; Pawliszyn, J. Development of a Hydrophilic Lipophilic Balanced Thin Film Solid Phase Microextraction Device for Balanced Determination of Volatile Organic Compounds. Anal. Chem. 2018, 90, 14072-14080.

(2) Grandy, J. J.; Boyaci, E.; Pawliszyn, J. Development of a Carbon Mesh Supported Thin Film Microextraction Membrane As a Means to Lower the Detection Limits of Benchtop and Portable GC/MS Instrumentation. Anal. Chem. 2016, 88 1760-1767

(3) Gómez-Ríos, G. A.; Pawliszyn, J. Development of Coated Blade Spray lonization Mass Spectrometry for the Quantitation of Target Analytes Present in Complex Matrices. Angew. Chemie - Int. Ed. 2014, 53 (52), 14503-14507.

(4) Boyaci, E.; Sparham, C.; Pawliszyn, J. Thin-Film Microextraction Coupled to LC-ESI-MS/MS for Determination of Quaternary Ammonium Compounds in Water Samples. Anal. Bioanal. Chem. 
2014, 406 (2), 409-420.

(5) Reyes-Garcés, N.; Gionfriddo, E.; Gómez-Ríos, G. A.; Alam, M. N.; Boyacl, E.; Bojko, B.; Singh, V.; Grandy, J.; Pawliszyn, J. Advances in Solid Phase Microextraction and Perspective on Future Directions. Anal. Chem. 2018, 90 (1), 302-360.

(6) Lashgari, M.; Singh, V.; Pawliszyn, J. A Critical Review on Regulatory Sample Preparation Methods: Validating Solid-Phase Microextraction Techniques. TrAC Trends Anal. Chem. 2019, 119, 115618.

(7) Musteata, M. L.; Musteata, F. M.; Pawliszyn, J. Biocompatible Solid-Phase Microextraction Coatings Based on Polyacrylonitrile and Solid-Phase Extraction Phases. Anal. Chem. 2007, 79 (18), 6903-6911.

(8) Bojko, B.; Cudjoe, E.; Gómez-Ríos, G. A.; Gorynski, K.; Jiang, R.; Reyes-Garcés, N.; Risticevic, S.; Silva, É. A. S.; Togunde, O.; Vuckovic, D.; et al. SPME - Quo Vadis? Anal. Chim. Acta 2012, 750, 132-151.

(9) Pawliszyn, J. Sample Preparation: Quo Vadis? Anal. Chem. 2003, 75 (11), 2543-2558.

(10) Feiring, A. E. Fluorine-Containing Polymers. In Encyclopedia of Materials: Science and Technology; Elsevier, 2001; pp 3209-3215.

(11) Lee, J. W.; Jung, J.; Cho, Y. H.; Yadav, S. K.; Baek, K. Y.; Park, H. B.; Hong, S. M.; Koo, C. M. FoulingTolerant Nanofibrous Polymer Membranes for Water Treatment. ACS Appl. Mater. Interfaces 2014, 6 (16), 14600-14607.

(12) Inan, T. Y.; Doan, H.; Unveren, E. E.; Eker, E. Sulfonated PEEK and Fluorinated Polymer Based Blends for Fuel Cell Applications: Investigation of the Effect of Type and Molecular Weight of the Fluorinated Polymers on the Membrane's Properties. Int. J. Hydrogen Energy 2010, 35 (21), 12038-12053.

(13) Wood, K. A.; Gaboury, S. R. Service Life Prediction of Colour Retention for PVDF Architectural Coatings with Organic Pigments. Surf. Coatings Int. Part B Coatings Trans. 2006, 89 (3), 231-235.

(14) Landry, V.; Blanchet, P. Weathering Resistance of Opaque PVDF-Acrylic Coatings Applied on Wood Substrates. Prog. Org. Coatings 2012, 75 (4), 494-501.

(15) Nandi, A. K.; Manna, S. Poly(Vinylidene Fluoride) Nanocomposites. In Encyclopedia of Polymer Science and Technology; John Wiley \& Sons, Inc.: Hoboken, NJ, USA, 2018; pp 1-43.

(16) Humphrey, J. S.; Amin-Sanayei, R. Vinylidene Fluoride Polymers. In Encyclopedia of Polymer Science and Technology; Wiley, 2003.

(17) Ameduri, B. From Vinylidene Fluoride (VDF) to the Applications of VDF-Containing Polymers and Copolymers: Recent Developments and Future Trends. Chem. Rev. 2009, 109 (12), 6632-6686.

(18) Laroche, G.; Marois, Y.; Guidoin, R.; King, M. W.; Martin, L.; How, T.; Douville, Y. Polyvinylidene Fluoride (PVDF) as a Biomaterial: From Polymeric Raw Material to Monofilament Vascular Suture. J. Biomed. Mater. Res. 1995, 29 (12), 1525-1536.

(19) Chen, W.; Zeng, J.; Chen, J.; Huang, X.; Jiang, Y.; Wang, Y.; Chen, X. High Extraction Efficiency for Polar Aromatic Compounds in Natural Water Samples Using Multiwalled Carbon Nanotubes/Nafion Solid-Phase Microextraction Coating. J. Chromatogr. A 2009, 1216 (52), 9143- 
9148.

(20) Zeng, J.; Chen, J.; Song, X.; Wang, Y.; Ha, J.; Chen, X.; Wang, X. An Electrochemically Enhanced Solid-Phase Microextraction Approach Based on a Multi-Walled Carbon Nanotubes/Nafion Composite Coating. J. Chromatogr. A 2010, 1217 (11), 1735-1741.

(21) Wu, M.; Zhang, H.; Zeng, B.; Zhao, F. A Strategy to Enhance the Antifouling Property of Coating for Direct Immersion Solid Phase Microextraction. J. Chromatogr. A 2015, 1384, 22-27.

(22) Martin, C. R.; Rhoades, T. A.; Ferguson, J. A. Dissolution of Perfluorinated lon Containing Polymers. Anal. Chem. 1982, 54 (9), 1639-1641.

(23) Gionfriddo, E.; Boyacl, E.; Pawliszyn, J. New Generation of Solid-Phase Microextraction Coatings for Complementary Separation Approaches: A Step toward Comprehensive Metabolomics and Multiresidue Analyses in Complex Matrices. Anal. Chem. 2017, 89 (7), 4046-4054.

(24) Zheng, M.; Kurt Wood; Gaboury, S. US7399533B2 POLYVNYLDENE FLUORIDE COATING FOR METAL SUBSTRATES FIELD OF THE INVENTION. 7399533B2, January 21, 2005.

(25) Liu, F.; Hashim, N. A.; Liu, Y.; Abed, M. R. M.; Li, K. Progress in the Production and Modification of PVDF Membranes. Journal of Membrane Science. 2011, 375, 1-27.

(26) Daems, N.; Milis, S.; Verbeke, R.; Szymczyk, A.; Pescarmona, P. P.; Vankelecom, I. F. J. HighPerformance Membranes with Full PH-Stability. RSC Adv. 2018, 8 (16), 8813-8827.

(27) Bottino, A.; Capannelli, G.; Munari, S.; Turturro, A. Solubility Parameters of Poly(Vinylidene Fluoride). J. Polym. Sci. Part B Polym. Phys. 1988, 26 (4), 785-794.

(28) Rajapandiyan, P.; Yang, J. Sensitive Cylindrical SERS Substrate Array for Rapid Microanalysis of Nucleobases. Anal. Chem. 2012, 84 (23), 10277-10282.

(29) Grandy, J. J.; Gómez-Ríos, G.; Pawliszyn, J. Development of a Standard Gas Generating Vial Comprised of a Silicon Oil-Polystyrene/Divinylbenzene Composite Sorbent. J. Chromatogr. A 2015, 1410, 1-8.

(30) Khaled, A.; Singh, V.; Pawliszyn, J. Comparison of Solid-Phase Microextraction to Solvent Extraction and QuEChERS for Quantitative Analysis of Veterinary Drug Residues in Chicken and Beef Matrices. J. Agric. Food Chem. 2019, 67 (46), 12663-12669.

(31) Jafari, Z.; Reza Hadjmohammadi, M. In situ growth of zeolitic imidazolate framework-8 on a GOPVDF membrane as a sorbent for thin-film microextraction of caffeine followed by quantitation through high-performance liquid chromatography Analyst 2020, 12, 1736--1743. 


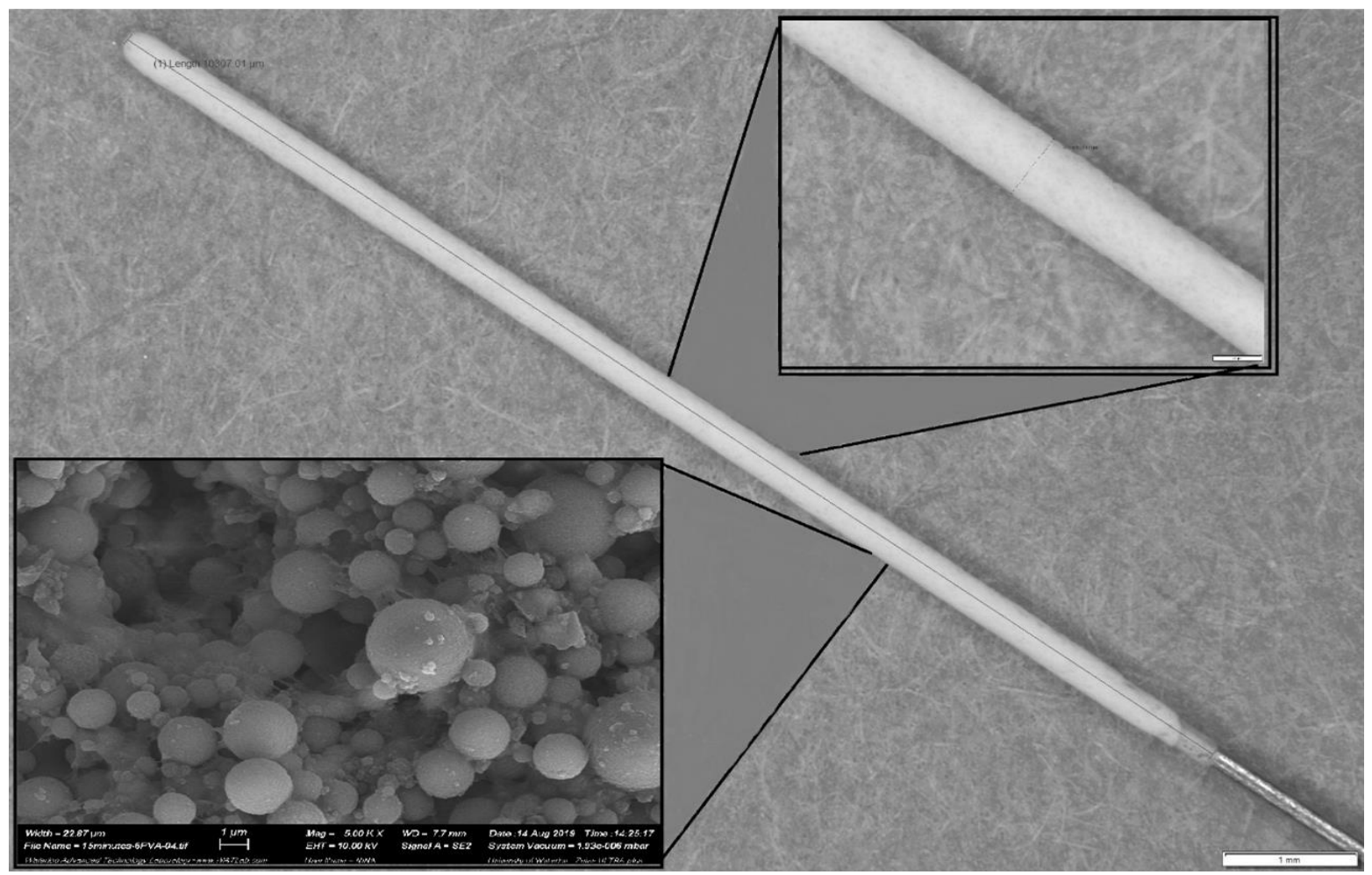

FIG. 1 


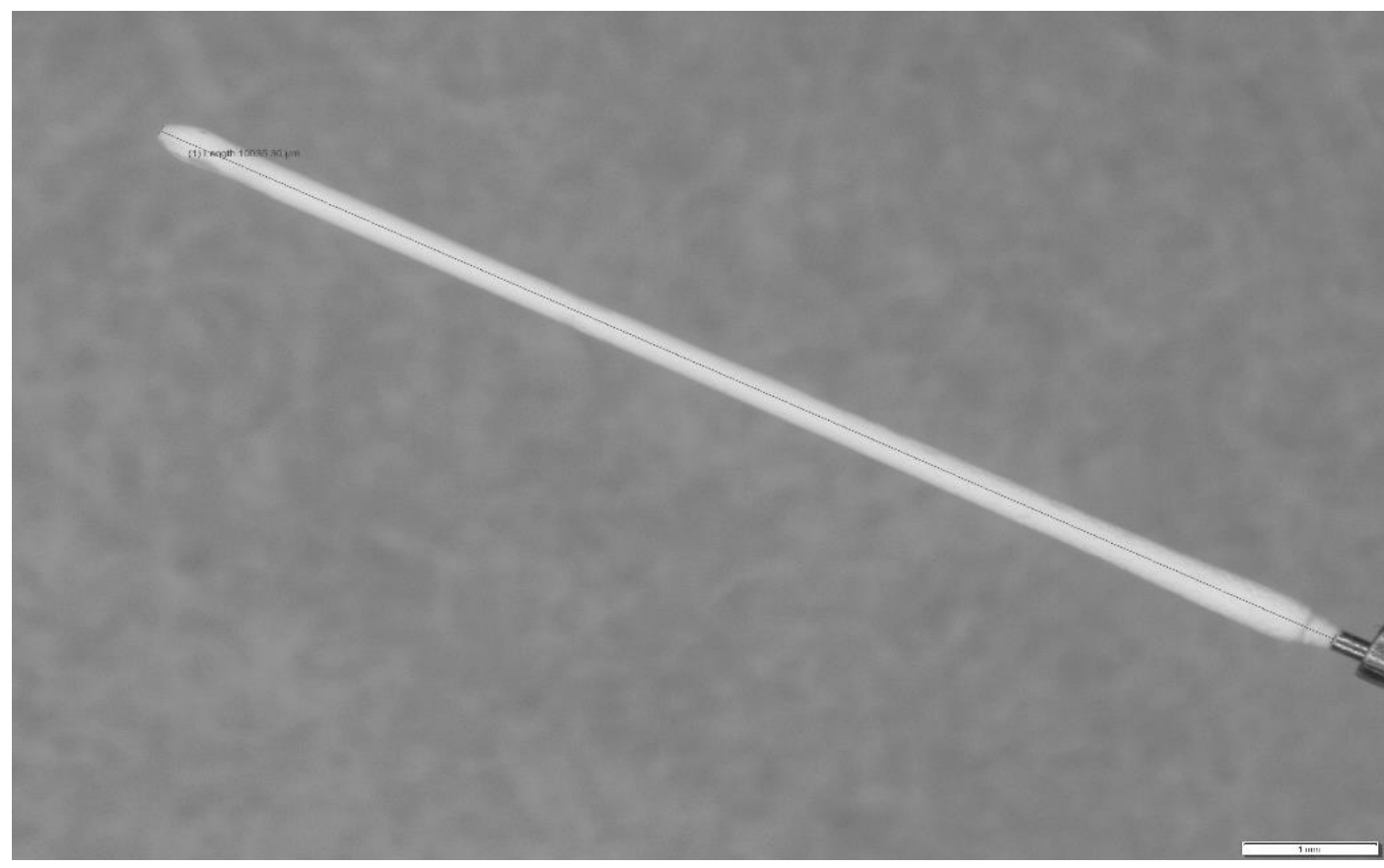

a

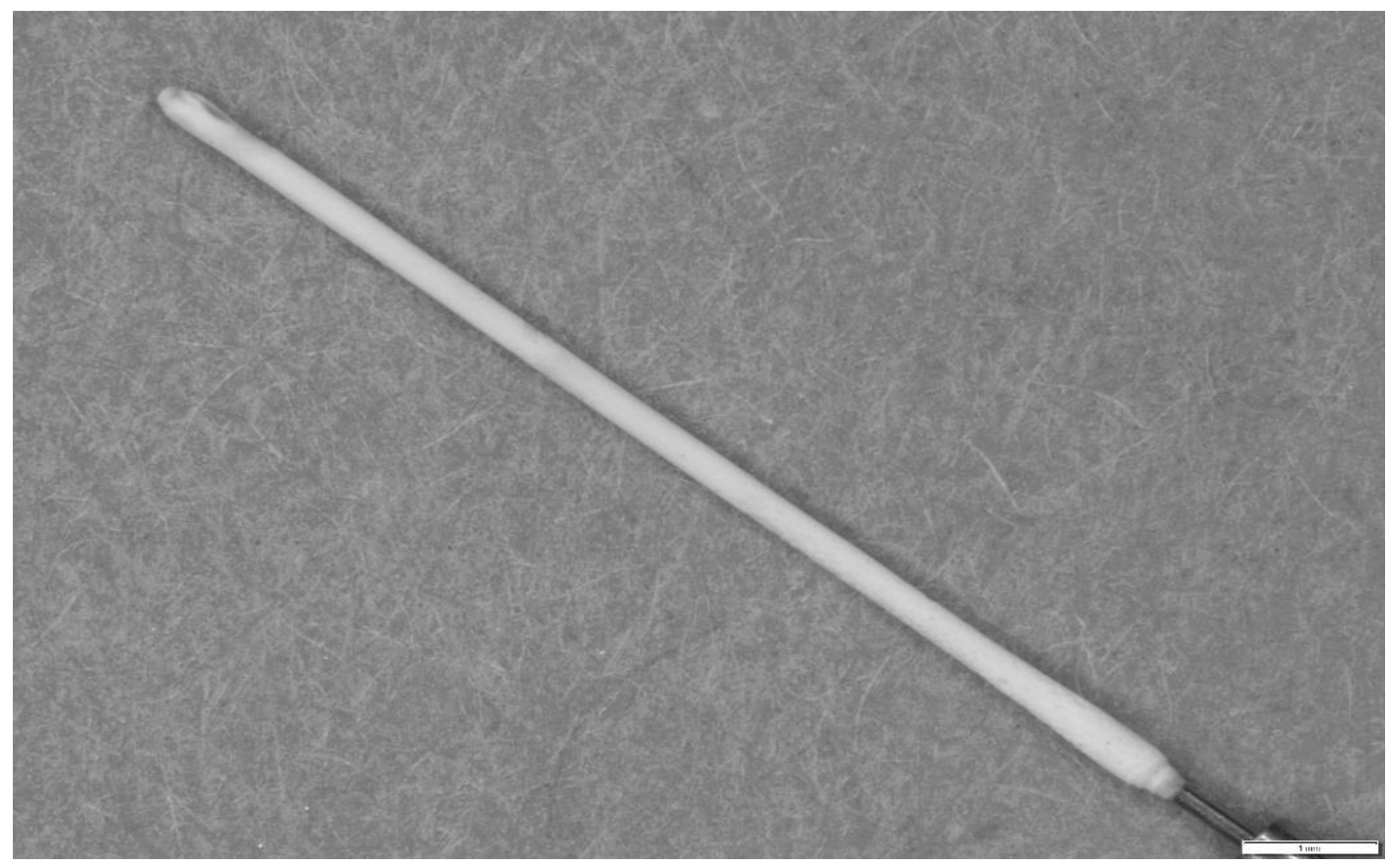

b

FIG. 2 


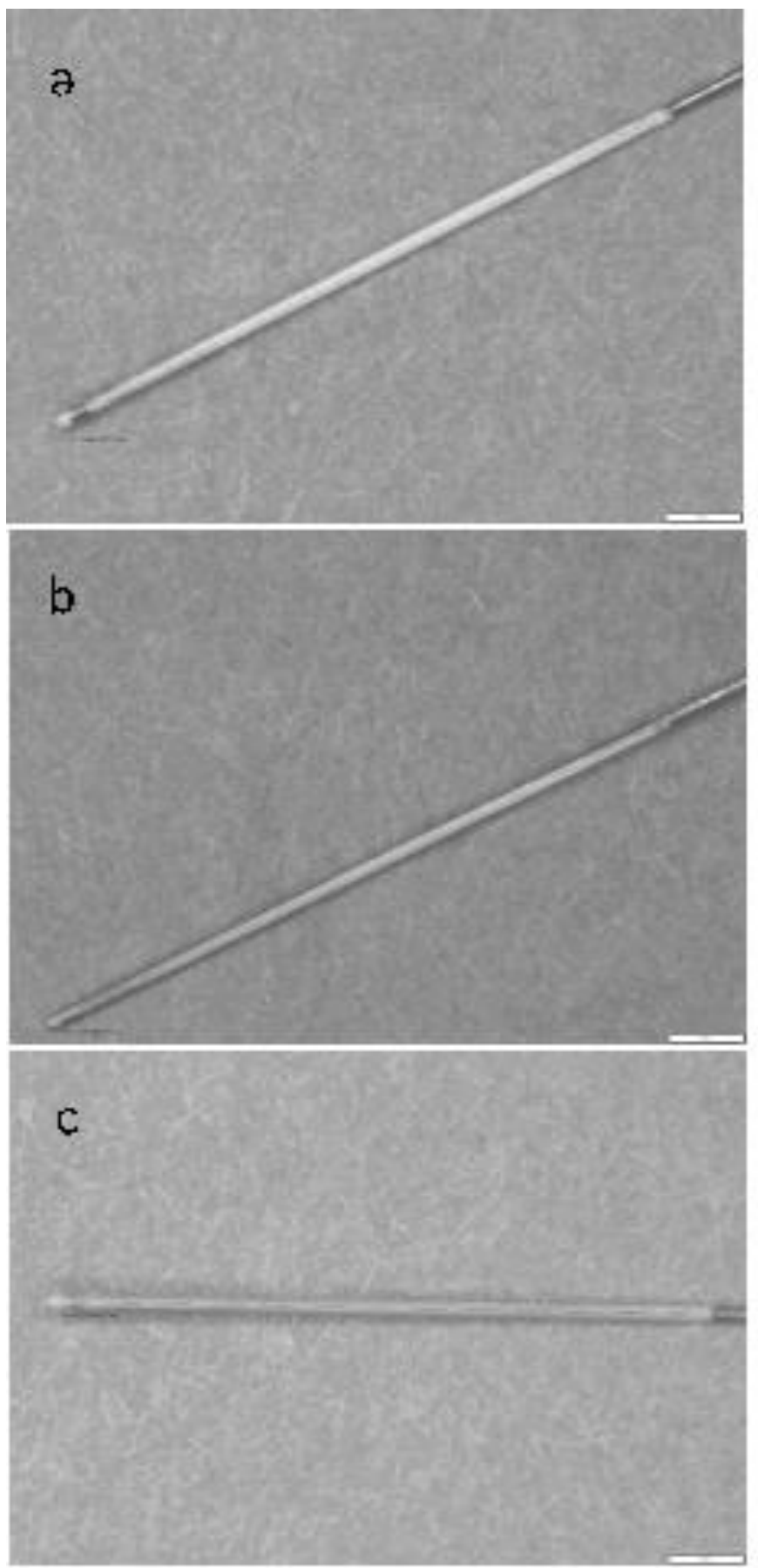

FIG. 3 
a

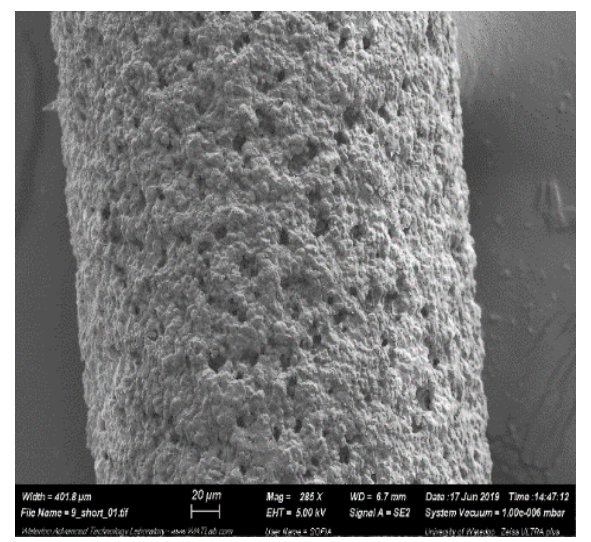

$12.5 \%$ PVDF, 180K MW

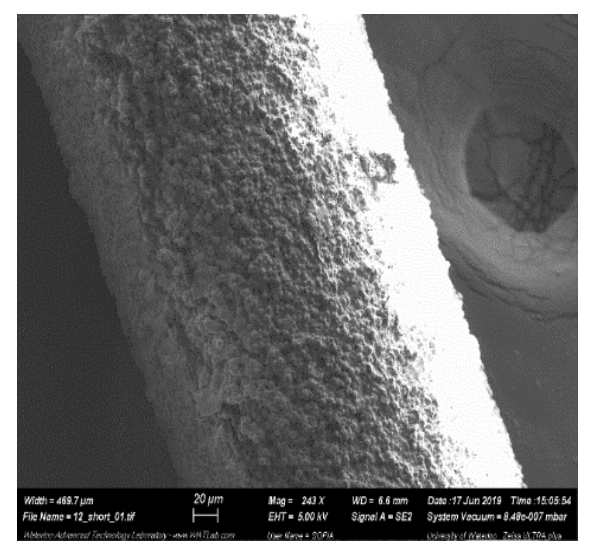

$16 \%$ PVDF, 275K MW

d b

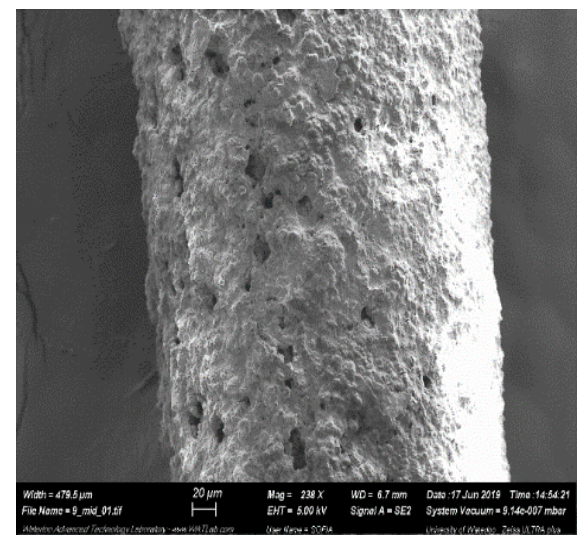

$16 \%$ PVDF, 180K MW

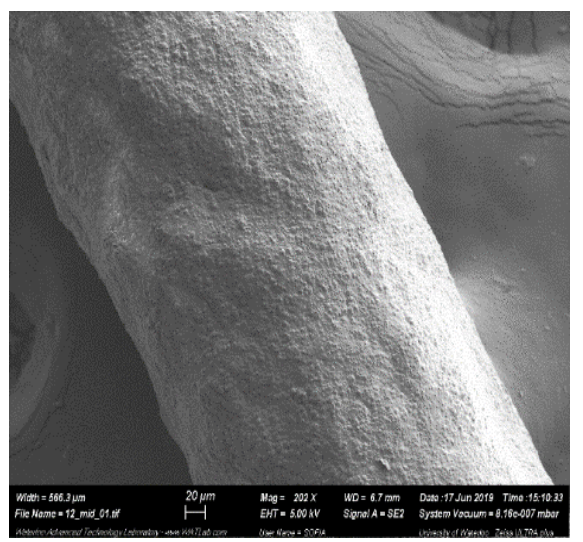

$18 \%$ PVDF, 275K MW

e

f

FIG. 4 

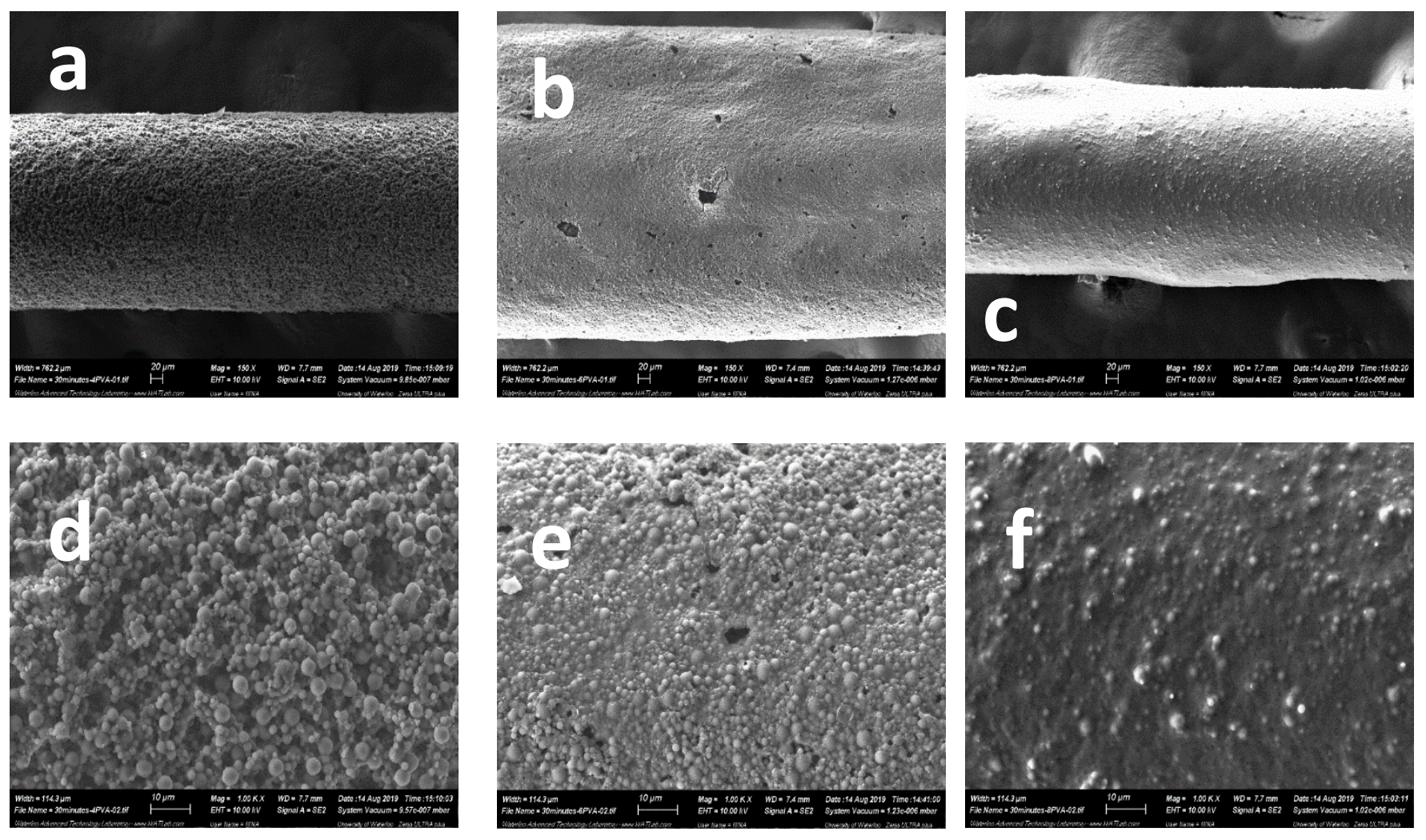

FIG. 5 

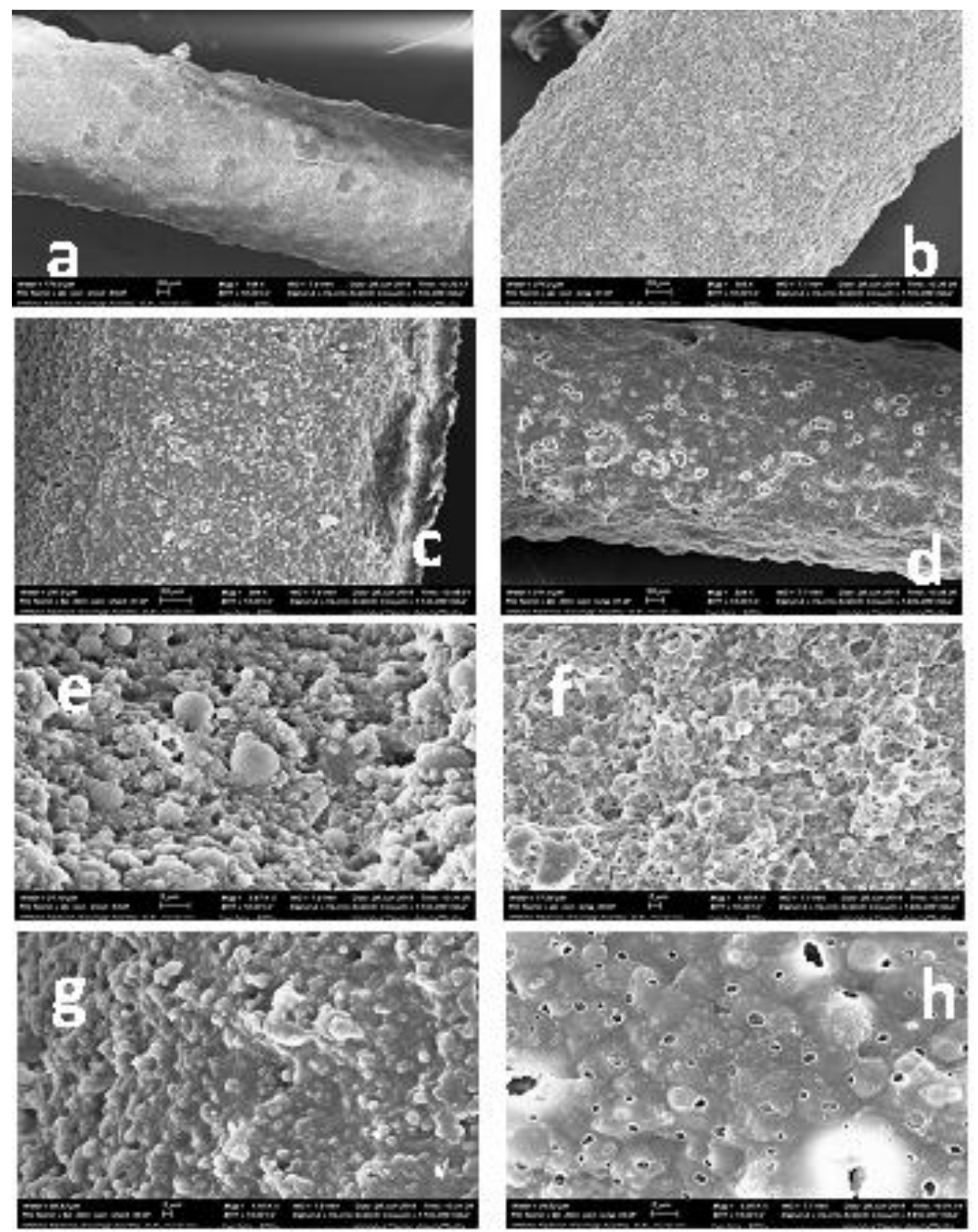

FIG. 6 


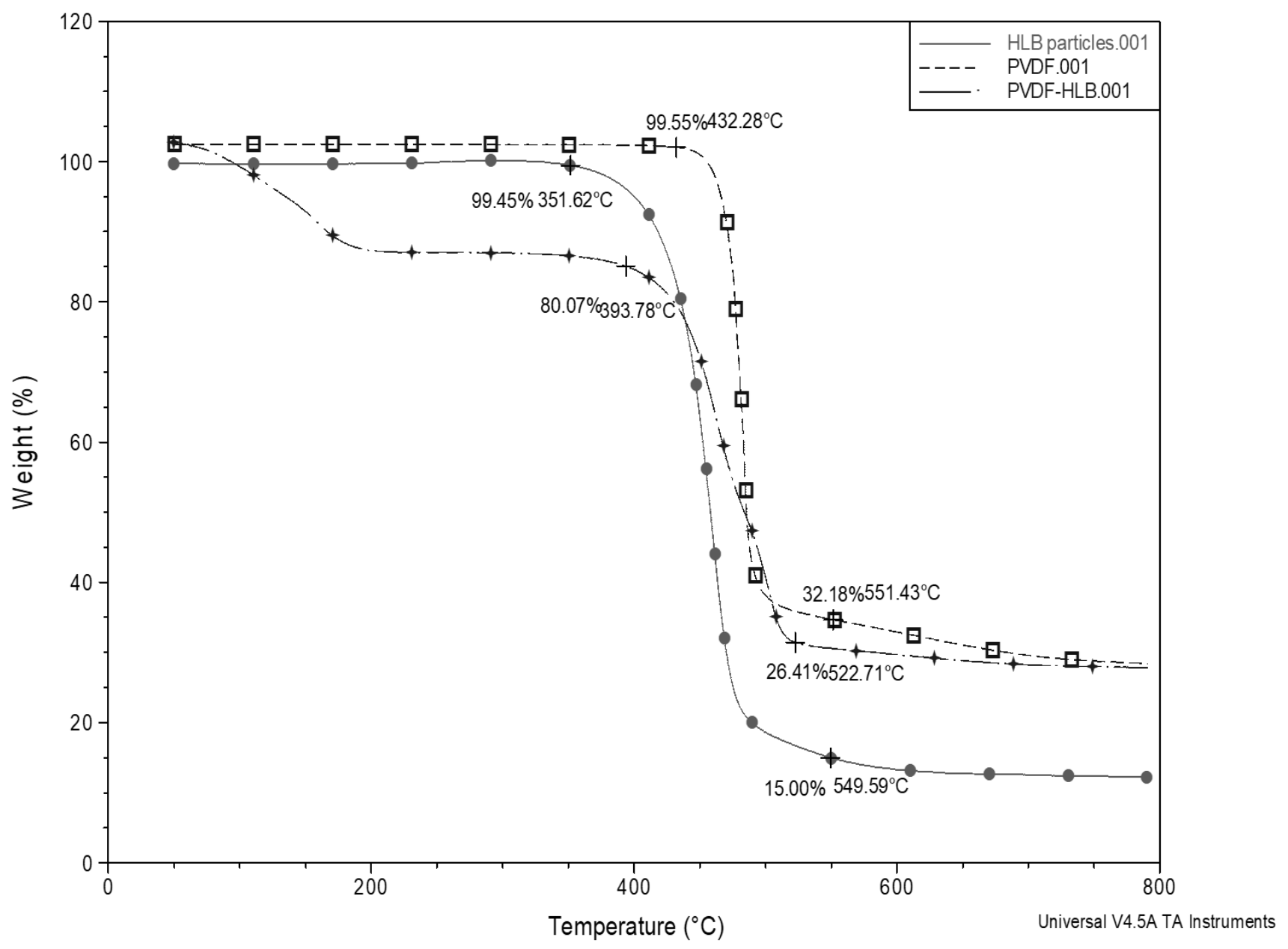

FIG. 7 


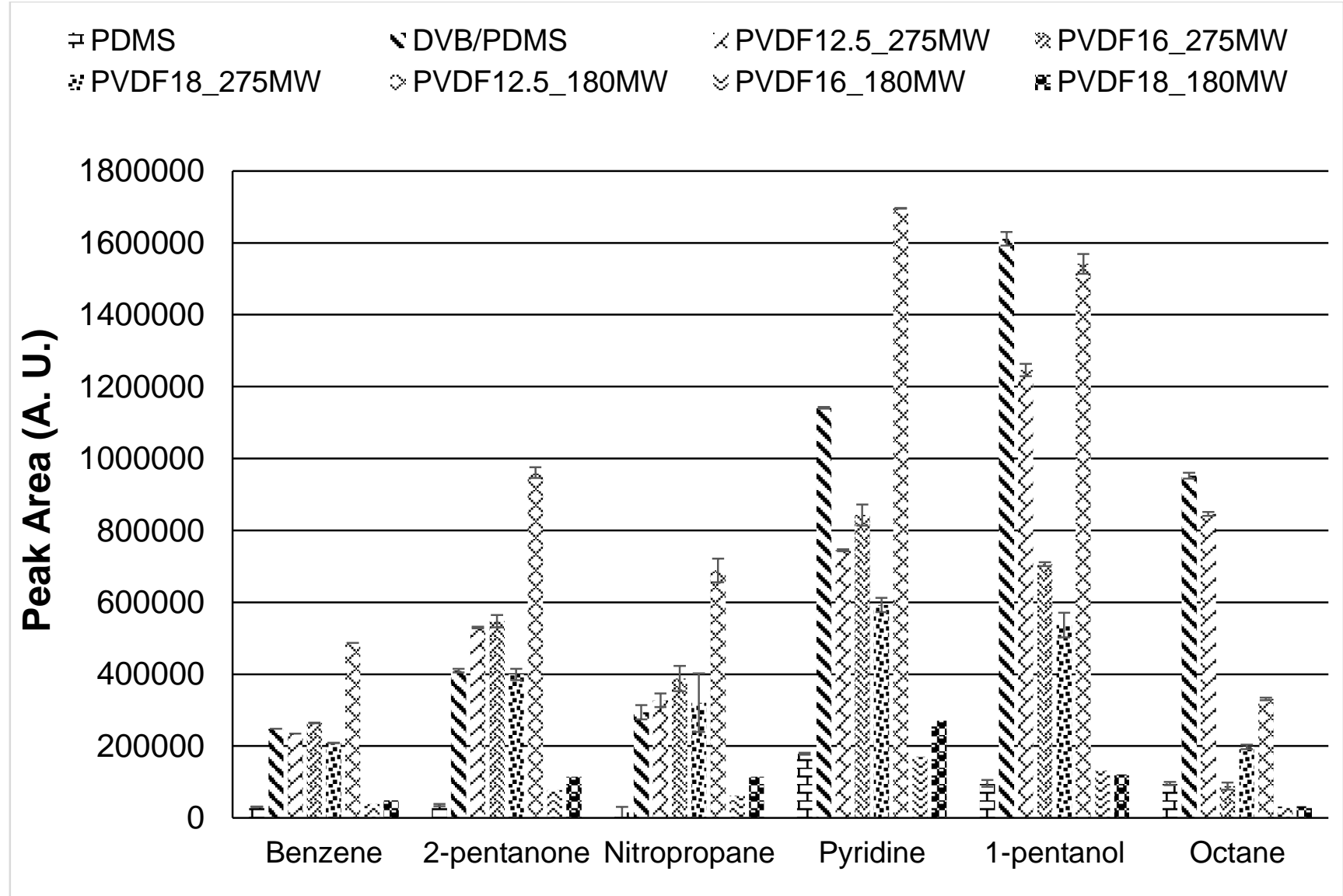

FIG. 8 


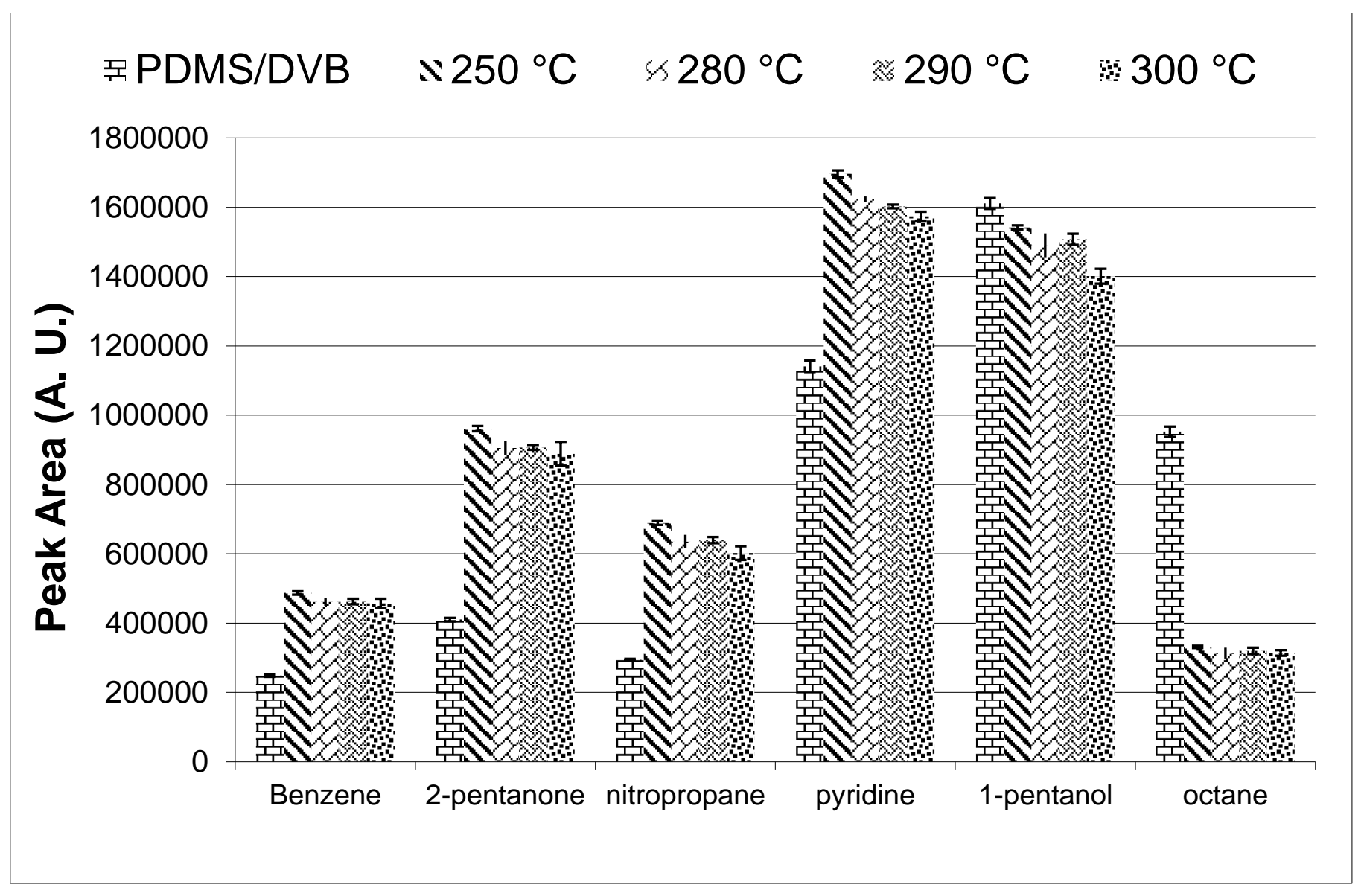

FIG. 9 


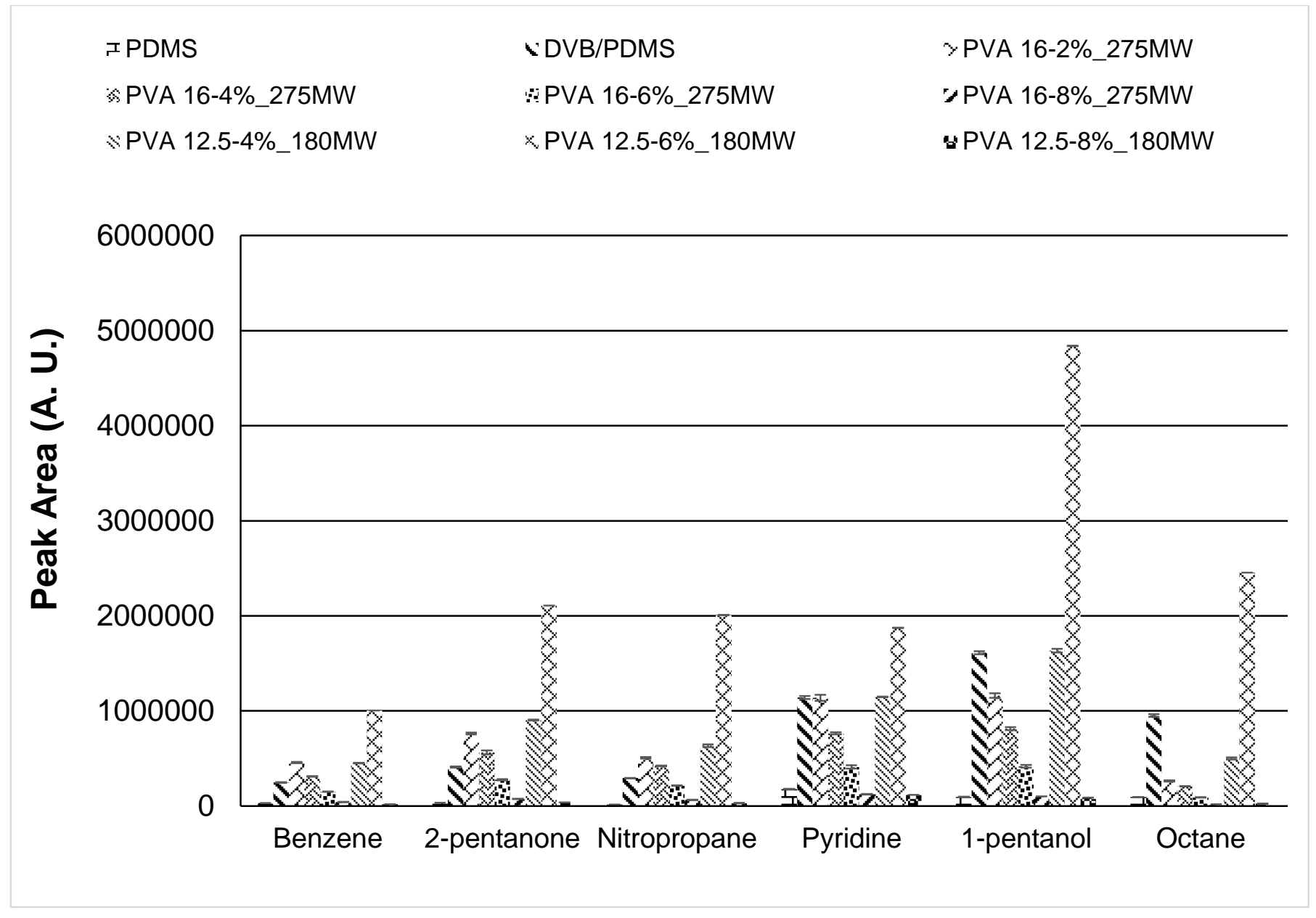

FIG. 10 


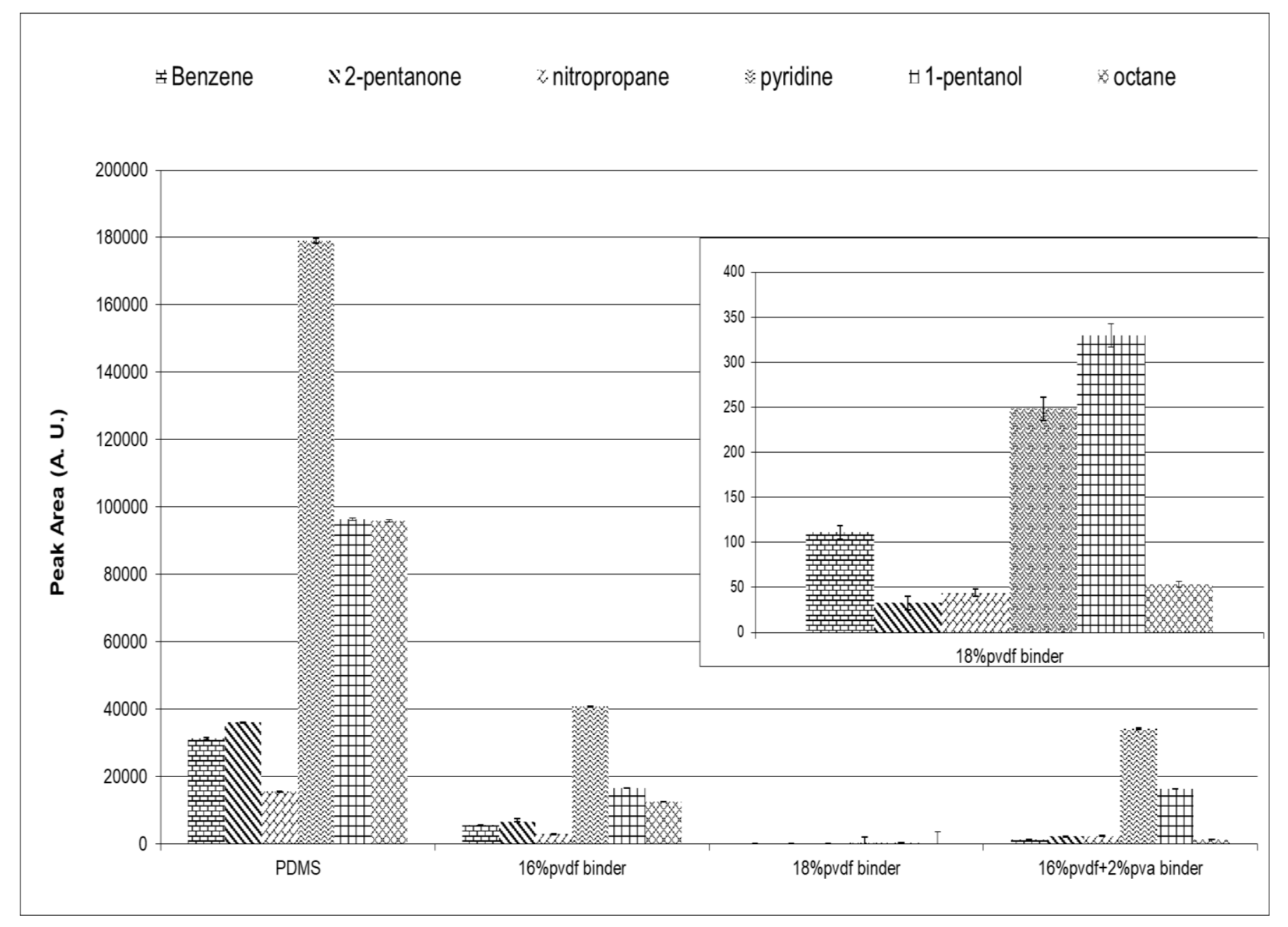

FIG. 11 


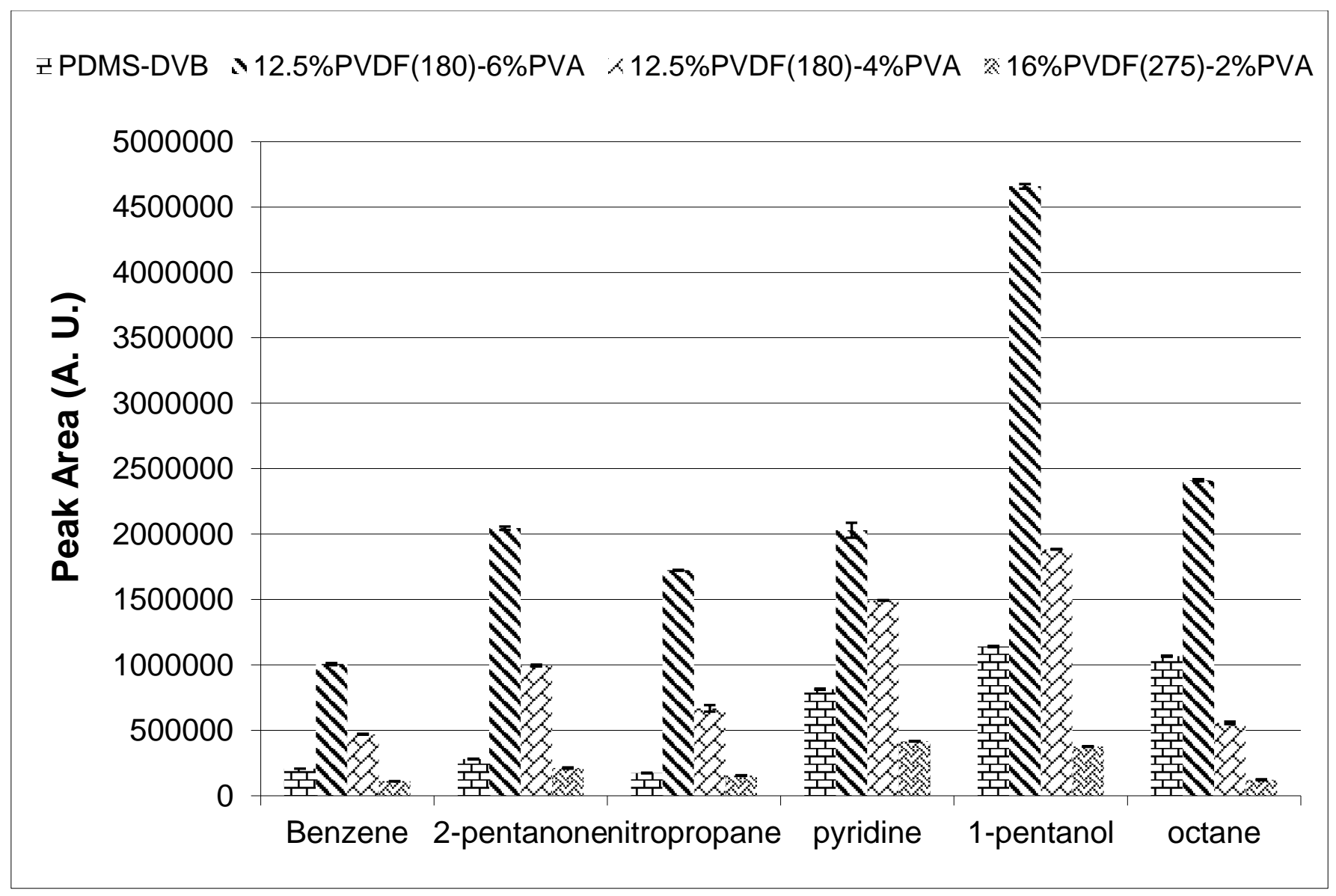

FIG. 12 


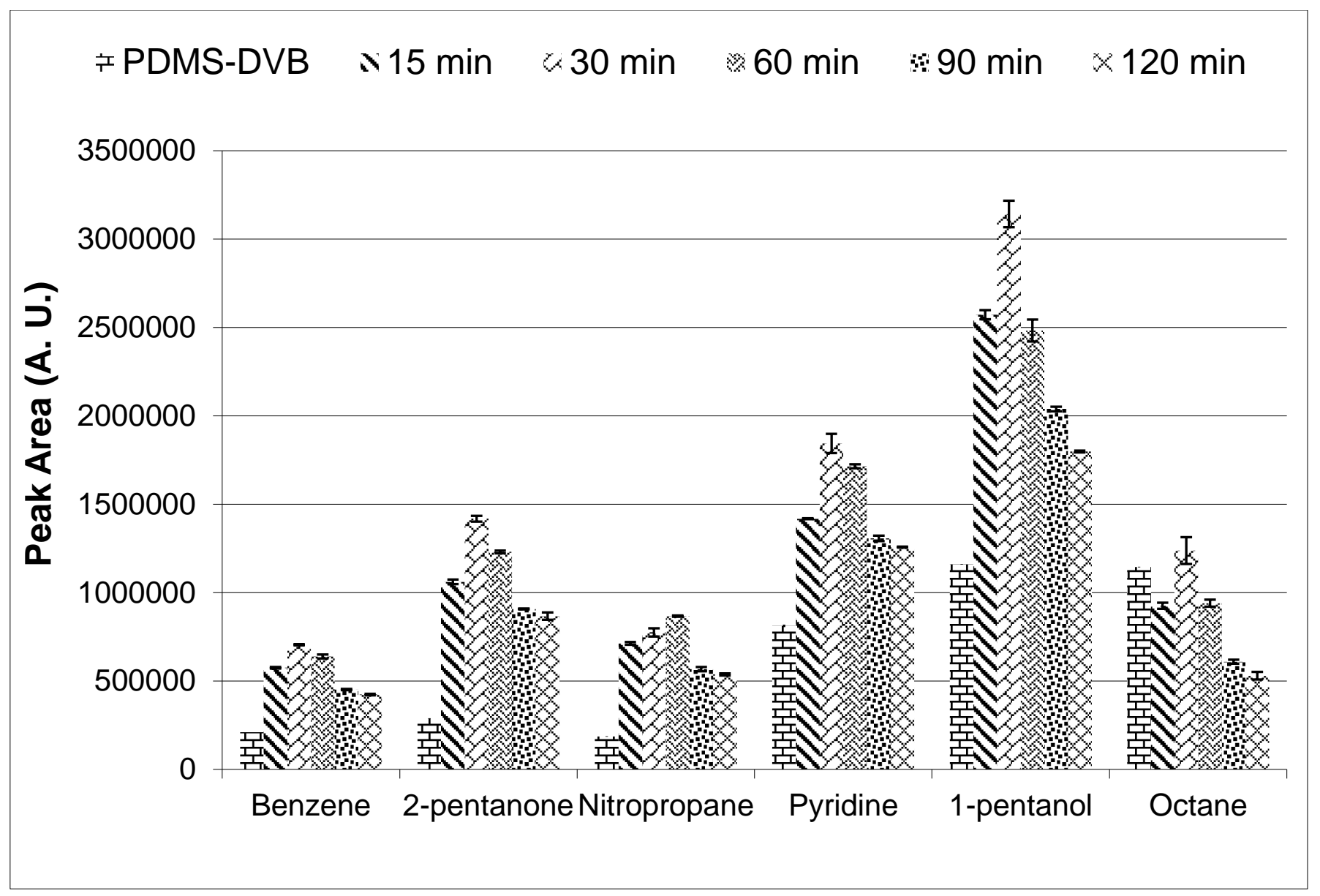

FIG. 13 


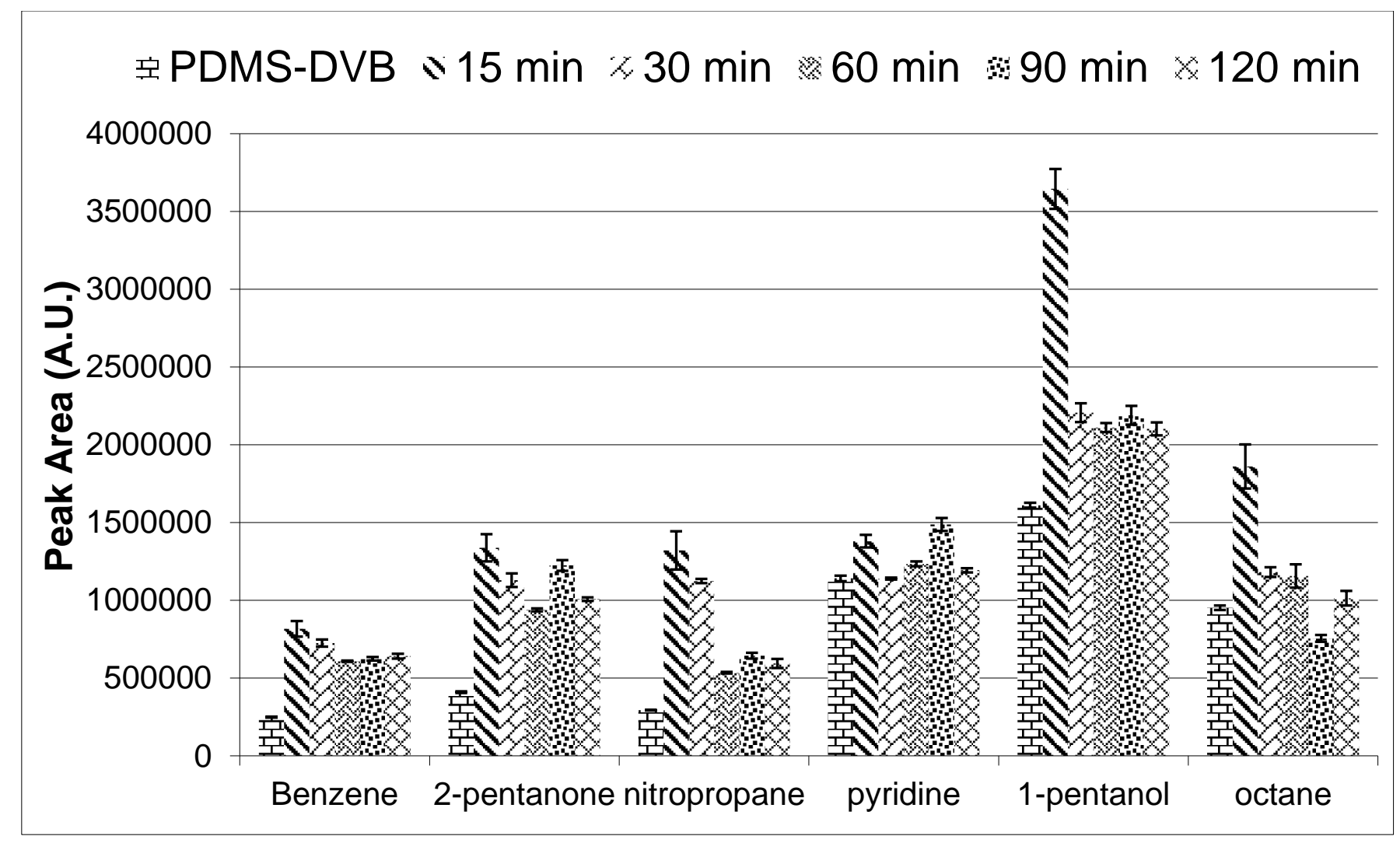

FIG. 14 


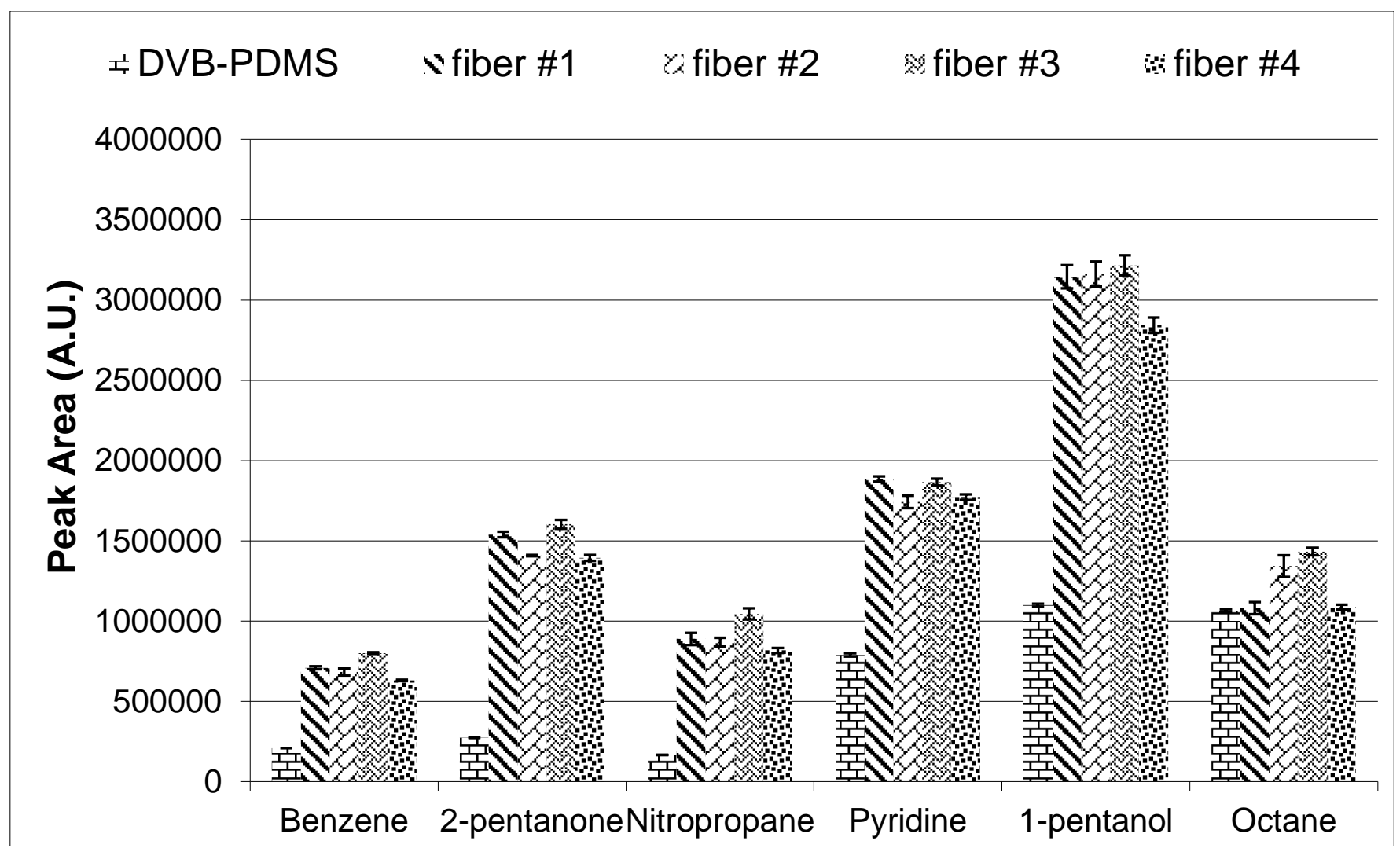

FIG. 15 

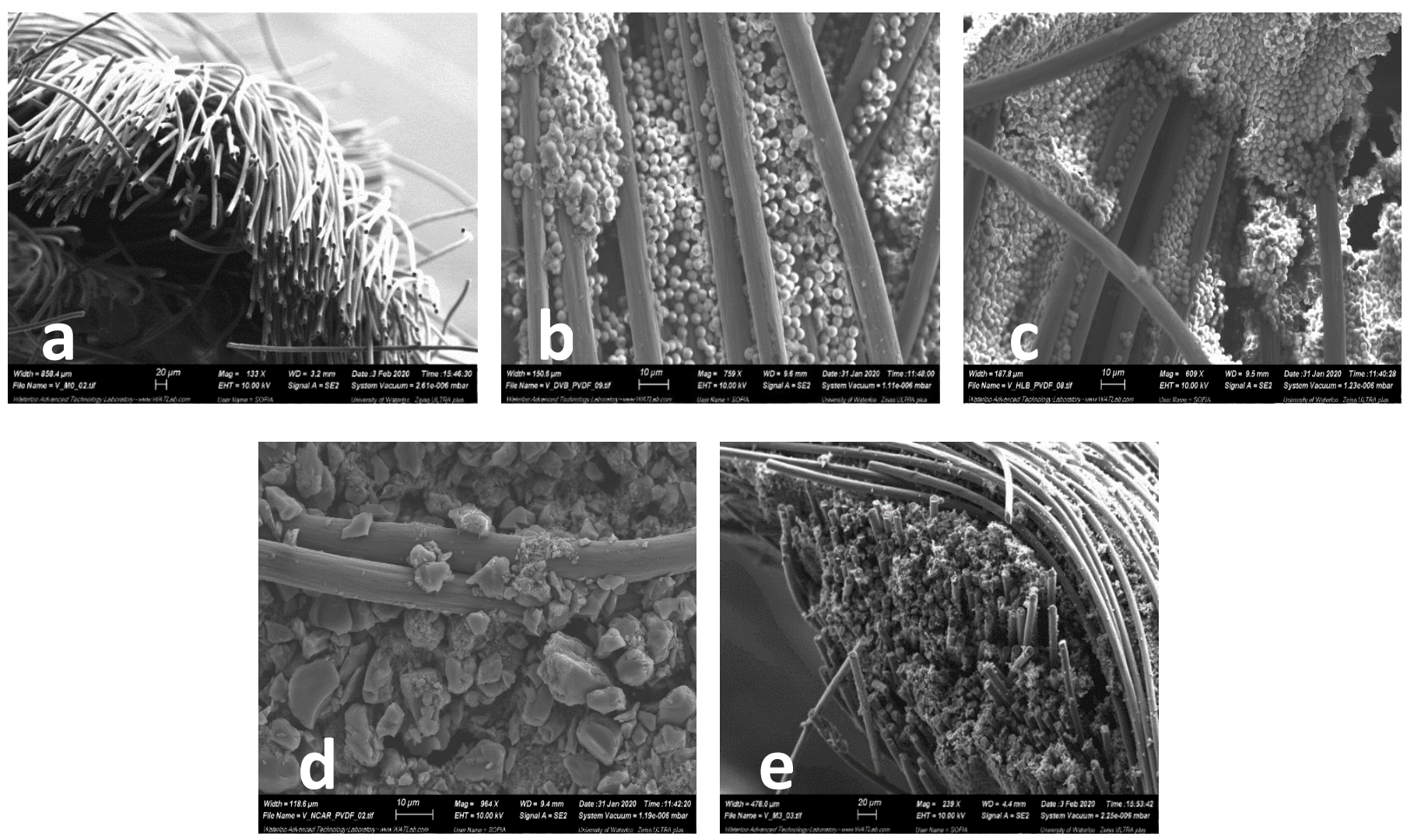

FIG. 16 


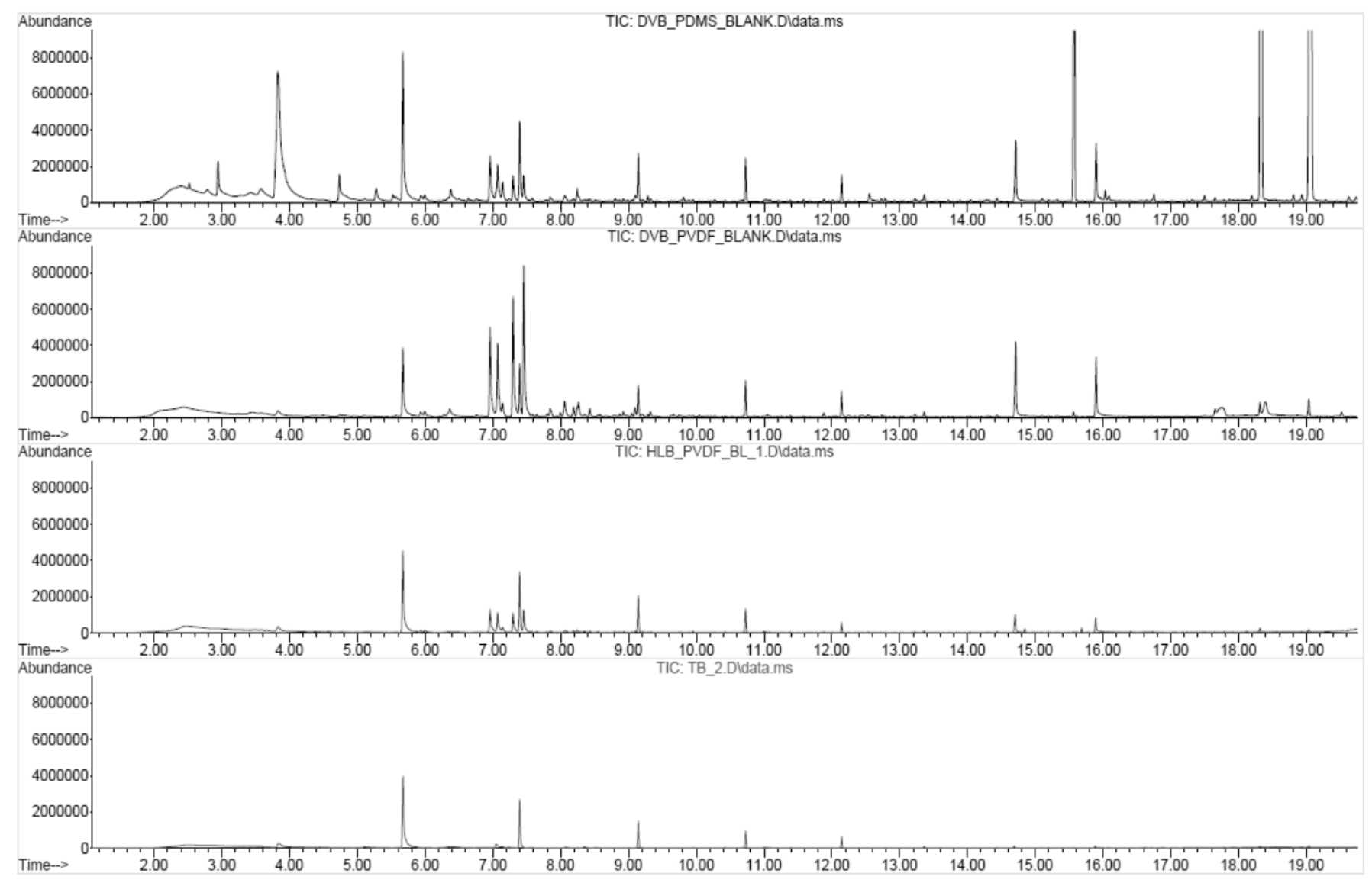

FIG. 17 

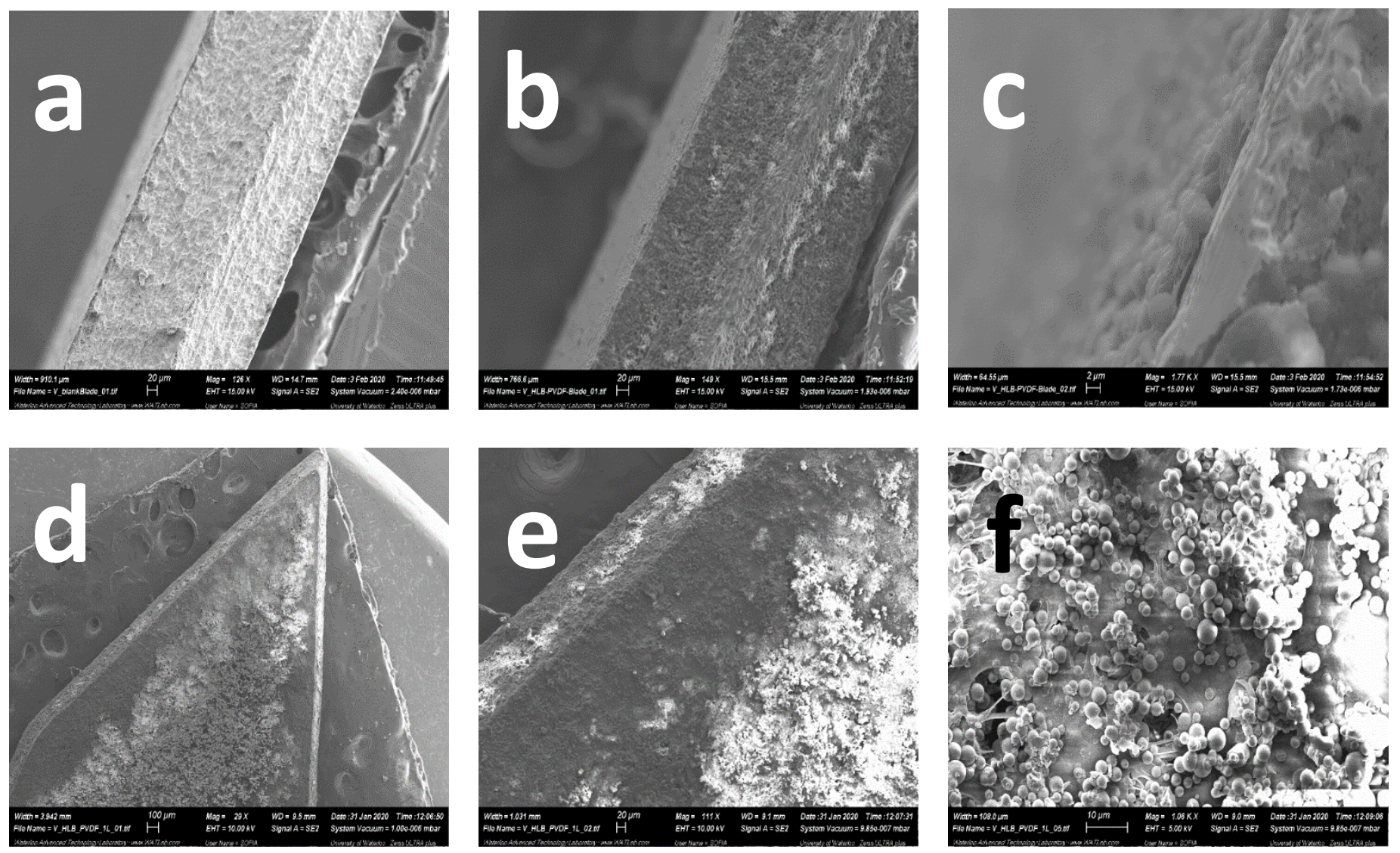

FIG. 18 


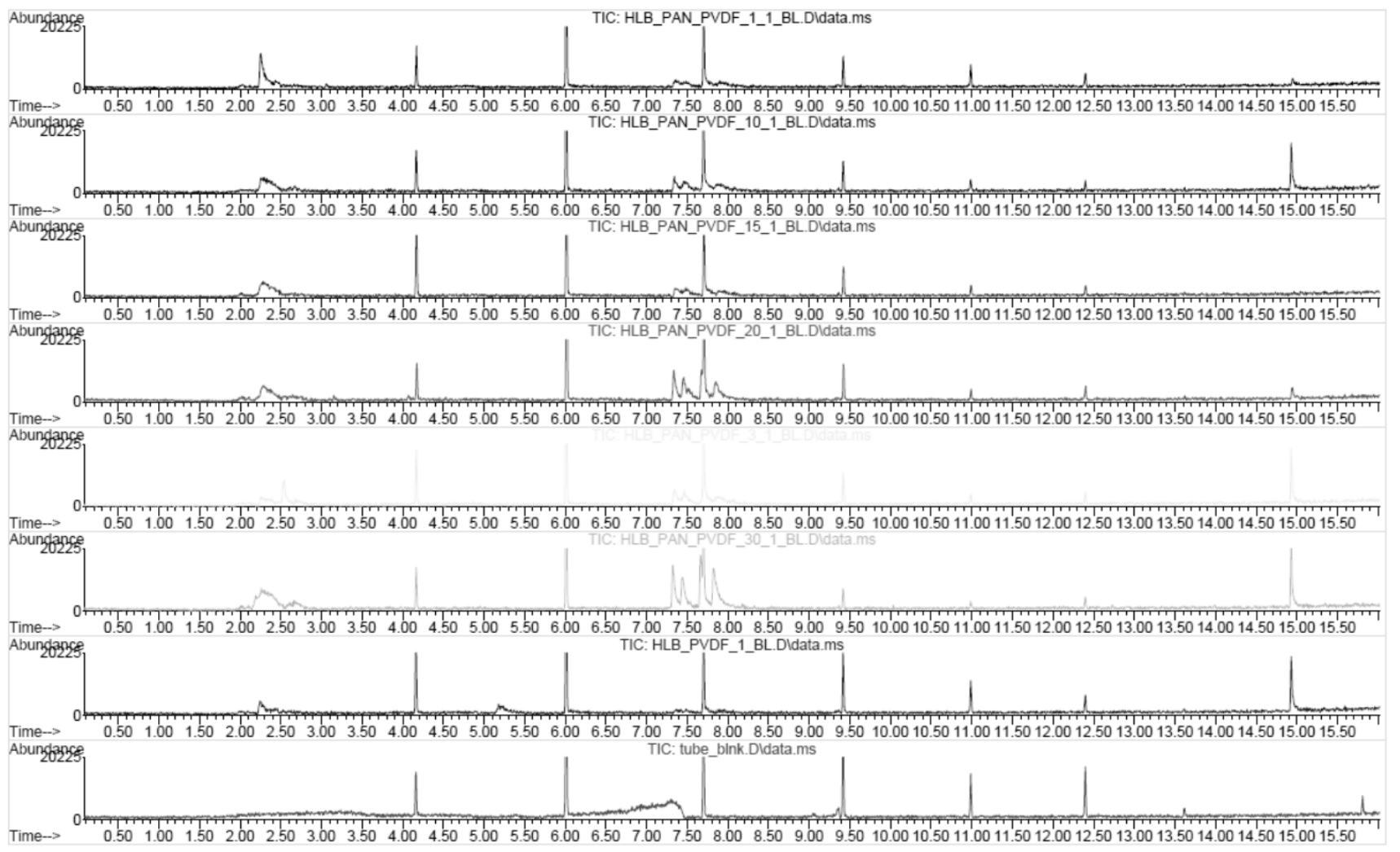

\section{FIG. 19}

